\title{
FAR-INFRARED FINE-STRUCTURE LINE DIAGNOSTICS OF ULTRALUMINOUS INFRARED GALAXIES
}

\author{
D. Farrah ${ }^{1}$, V. Lebouteiller ${ }^{2,3}$, H. W. W. Spoon ${ }^{2}$, J. Bernard-Salas ${ }^{4}$, C. Pearson ${ }^{4,5}$, D. Rigopoulou ${ }^{5,6}$, H. A. SMith $^{7}$, \\ E. González-Alfonso ${ }^{8}$, D. L. Clements ${ }^{9}$, A. Efstathiou ${ }^{10}$, D. Cormier ${ }^{11}$, J. Afonso ${ }^{12,13}$, S. M. Petty ${ }^{1}$, K. Harris ${ }^{1}$, \\ P. Hurley ${ }^{14}$, C. Borys ${ }^{15}$, A. Verma ${ }^{6}$, A. Cooray ${ }^{16}$, And V. Salvatelli ${ }^{16,17}$ \\ ${ }^{1}$ Department of Physics, Virginia Tech, Blacksburg, VA 24061, USA \\ ${ }^{2}$ Cornell University, CRSR, Space Sciences Building, Ithaca, NY 14853, USA \\ ${ }^{3}$ CEA-Saclay, DSM/IRFU/SAp, F-91191 Gif-sur-Yvette, France \\ ${ }^{4}$ Department of Physics \& Astronomy, The Open University, Milton Keynes MK7 6AA, UK \\ ${ }^{5}$ RAL Space, Rutherford Appleton Laboratory, Harwell, Oxford OX11 0QX, UK \\ ${ }^{6}$ Oxford Astrophysics, Denys Wilkinson Building, University of Oxford, Keble Rd, Oxford OX1 3RH, UK \\ ${ }^{7}$ Harvard-Smithsonian Center for Astrophysics, 60 Garden Street, Cambridge, MA 02138, USA \\ ${ }^{8}$ Universidad de Alcalá, Departamento de Física y Matemáticas, Campus Universitario, E-28871 Alcalá de Henares, Madrid, Spain \\ ${ }^{9}$ Physics Department, Imperial College London, Prince Consort Road, London SW7 2AZ, UK \\ ${ }^{10}$ School of Sciences, European University Cyprus, Diogenes Street, Engomi, 1516 Nicosia, Cyprus \\ ${ }^{11}$ Institut für theoretische Astrophysik, Zentrum für Astronomie der Universität Heidelberg, Albert-Ueberle Str. 2, D-69120 Heidelberg, Germany \\ ${ }^{12}$ Centro de Astronomia e Astrofísica da Universidade de Lisboa, Observatório Astronómico de Lisboa, Tapada da Ajuda, 1349-018 Lisbon, Portugal \\ ${ }^{13}$ Department of Physics, Faculty of Sciences, University of Lisbon, Campo Grande, 1749-016 Lisbon, Portugal \\ ${ }^{14}$ Department of Physics \& Astronomy, University of Sussex, Falmer, Brighton BN1 9QH, UK \\ ${ }^{15}$ Infrared Processing and Analysis Center, MS220-6, California Institute of Technology, Pasadena, CA 91125, USA \\ ${ }^{16}$ Department of Physics \& Astronomy, University of California, Irvine, CA 92697, USA \\ ${ }^{17}$ Physics Department and INFN, Università di Roma "La Sapienza," Ple Aldo Moro 2, I-00185, Rome, Italy \\ Received 2013 May 8; accepted 2013 August 6; published 2013 September 24
}

\begin{abstract}
We present Herschel observations of 6 fine-structure lines in 25 ultraluminous infrared galaxies at $z<0.27$. The lines, [O III] $52 \mu \mathrm{m},\left[\mathrm{N}\right.$ III] $57 \mu \mathrm{m},\left[\mathrm{O}_{\mathrm{I}}\right] 63 \mu \mathrm{m}$, [N II] $122 \mu \mathrm{m}$, [O I] $145 \mu \mathrm{m}$, and [C II] $158 \mu \mathrm{m}$, are mostly single Gaussians with widths $<600 \mathrm{~km} \mathrm{~s}^{-1}$ and luminosities of $10^{7}-10^{9} L_{\odot}$. There are deficits in the $[\mathrm{O}$ I $] 63 / L_{\mathrm{IR}}$, $[\mathrm{N} \mathrm{II}] / L_{\mathrm{IR}},[\mathrm{O} \mathrm{I}] 145 / L_{\mathrm{IR}}$, and $[\mathrm{CII}] / L_{\mathrm{IR}}$ ratios compared to lower luminosity systems. The majority of the line deficits are consistent with dustier $\mathrm{H}$ II regions, but part of the [C $\mathrm{II}]$ deficit may arise from an additional mechanism, plausibly charged dust grains. This is consistent with some of the [C II] originating from photodissociation regions or the interstellar medium (ISM). We derive relations between far-IR line luminosities and both the IR luminosity and star formation rate. We find that [N II] and both [O I] lines are good tracers of the IR luminosity and star formation rate. In contrast, $[\mathrm{C} \mathrm{II}]$ is a poor tracer of the IR luminosity and star formation rate, and does not improve as a tracer of either quantity if the $[\mathrm{C} \mathrm{II}]$ deficit is accounted for. The continuum luminosity densities also correlate with the IR luminosity and star formation rate. We derive ranges for the gas density and ultraviolet radiation intensity of $10^{1}<n<10^{2.5}$ and $10^{2.2}<G_{0}<10^{3.6}$, respectively. These ranges depend on optical type, the importance of star formation, and merger stage. We do not find relationships between far-IR line properties and several other parameters: active galactic nucleus (AGN) activity, merger stage, mid-IR excitation, and SMBH mass. We conclude that these far-IR lines arise from gas heated by starlight, and that they are not strongly influenced by AGN activity.
\end{abstract}

Key words: galaxies: active - galaxies: evolution - galaxies: ISM - galaxies: starburst - infrared: galaxies

Online-only material: color figures

\section{INTRODUCTION}

Ultraluminous infrared galaxies (ULIRGS, objects with $L_{\mathrm{IR}}>10^{12} L_{\odot}$; Sanders \& Mirabel 1996; Lonsdale et al. 2006) are a cosmologically important population whose nature changes substantially with redshift. At $z<0.3$, ULIRGs are rare (e.g., Soifer \& Neugebauer 1991; Vaccari et al. 2010), with less than one per $\sim$ hundred square degrees. They are invariably mergers between approximately equal mass galaxies (Clements et al. 1996; Surace et al. 2000; Cui et al. 2001; Farrah et al. 2001; Bushouse et al. 2002; Veilleux et al. 2002, 2006). Evidence suggests that their IR emission arises mainly from high rates of star formation (Genzel et al 1998; Tran et al. 2001; Franceschini et al. 2003; Nardini et al. 2010; Wang et al. 2011), though on the order of half of them also contain a luminous active galactic nucleus (AGN; Rigopoulou et al. 1999; Farrah et al. 2003; Imanishi et al. 2007; Vega et al. 2008; Nardini \& Risaliti 2011). The AGNs in ULIRGs may become more impor- tant with increasing IR luminosity and advancing merger stage (Teng \& Veilleux 2010; Yuan et al. 2010; Stierwalt et al. 2013), and sometimes initiate powerful outflows (Spoon et al. 2009; Fischer et al. 2010; Rupke \& Veilleux 2011; Sturm et al. 2011; Westmoquette et al. 2012; Rodríguez Zaurín et al. 2013). A small fraction of (low-redshift) ULIRGs become optical QSOs (Tacconi et al. 2002; Kawakatu et al. 2006, 2007; Farrah et al. 2007b; Meng et al. 2010; Hou et al. 2011), and a large fraction end up as early-type galaxies (Genzel et al. 2001; Dasyra et al. 2006; Rothberg et al. 2013; Wang et al. 2013).

Over $0.3 \lesssim z<1$, the number of ULIRGs rises rapidly (e.g., Le Floc'h et al. 2005), reaching a density on the sky of several hundred per square degree at $z \gtrsim 1$ (Rowan-Robinson et al. 1997; Dole et al. 2001; Borys et al. 2003; Mortier et al. 2005; Austermann et al. 2010; Goto et al. 2011). The fraction of $z \gtrsim 1$ ULIRGs that are starburst-dominated mergers is high (Farrah et al. 2002; Chapman et al. 2003; Smail et al. 2004; Takata et al. 2006; Borys et al. 2006; Valiante et al. 2007; Berta et al. 2007; 
Bridge et al. 2007; Lonsdale et al. 2009; Huang et al. 2009; Magnelli et al. 2012; Lo Faro et al. 2013; Johnson et al. 2013) but the merger fraction may be lower than locally (Melbourne et al. 2008; Kartaltepe et al. 2010; Draper \& Ballantyne 2012, but see also Xu et al. 2012). High redshift ULIRGs may also have a wider range in dust temperature (Magdis et al. 2010; RowanRobinson et al. 2010; Symeonidis et al. 2011, 2013; Bridge et al. 2013; Hwang et al. 2010) and spectral energy distribution shapes (Farrah et al. 2008; Sajina et al. 2012; Nordon et al. 2012), and a greater star formation efficiency (Iglesias-Páramo et al. 2007; Combes et al. 2011, 2013; Hanami et al. 2012; Geach et al. 2013) compared to local examples.

Determining why the number and properties of ULIRGs change so markedly with redshift may provide insight into the history of stellar and SMBH mass assembly in $\gtrsim L^{*}$ galaxies. ULIRGs at $z<0.3$ are central to this endeavor, as they establish a baseline from which to measure evolution with redshift in the ULIRG population. The far-infrared $(\simeq 50-500 \mu \mathrm{m})$ is a powerful tool for studying ULIRGs, as demonstrated by the Infrared Space Observatory (ISO; e.g., Fischer et al. 1999; Negishi et al. 2001; Luhman et al. 2003; Spinoglio et al. 2005; Brauher et al. 2008). The Herschel Space Observatory (Pilbratt et al. 2010) offers dramatic advances in far-infrared observing capability over ISO. Its instruments, the Photodetector Array Camera and Spectrometer (PACS; Poglitsch et al. 2010), Spectral and Photometric Imaging REceiver (SPIRE; Griffin et al. 2010), and Heterodyne Instrument for the Far Infrared (de Graauw et al. 2010) can observe wavelength ranges that are inaccessible from the ground and have improved sensitivity and resolution over previous space-based facilities.

We have used Herschel to conduct the Herschel ULIRG Survey (HERUS), which assembles PACS and SPIRE observations of nearly all ULIRGs with a $60 \mu \mathrm{m}$ flux greater than $\sim 1.7 \mathrm{Jy}$. In this paper, we present observations of fine-structure lines for 24 objects of the sample. An analysis of the SPIRE FTS spectra is presented in C. Pearson et al. (2013, in preparation). Observations of the $\mathrm{OH} 119 \mu \mathrm{m}$ and $79 \mu \mathrm{m}$ profiles are presented in Spoon et al. (2013), while modeling of these profiles is presented in H. A. Smith et al. (in preparation). Finally, a detailed study of the ULIRG IRAS 08572+3915 is presented in Efstathiou et al. (2013). We define infrared luminosity, $L_{\mathrm{IR}}$, to be the luminosity integrated over $8-1000 \mu \mathrm{m}$ in the rest frame. We quote luminosities and masses in units of Solar $\left(L_{\odot}=3.839 \times 10^{26}\right.$ Watts, $M_{\odot}=1.99 \times 10^{30} \mathrm{Kg}$, respectively). We assume a spatially flat cosmology with $H_{0}=67.3 \mathrm{~km} \mathrm{~s}^{-1} \mathrm{Mpc}^{-1}, \Omega=1$, and $\Omega_{m}=0.315$ (Planck Collaboration et al. 2013).

\section{METHODS}

\subsection{Sample Selection}

HERUS is a photometric and spectroscopic atlas of the $z<$ 0.27 ULIRG population. The sample comprises all 40 ULIRGs from the IRAS PSC- $z$ survey (Saunders et al. 2000) with $60 \mu \mathrm{m}$ fluxes greater than $2 \mathrm{Jy}$, together with three randomly selected ULIRGs with lower $60 \mu \mathrm{m}$ fluxes: IRAS 00397-1312 (1.8 Jy), IRAS 07598+6508 (1.7 Jy), and IRAS 13451+1232 (1.9 Jy). All objects have been observed with the Infrared Spectrograph (IRS; Houck et al. 2004) on-board Spitzer (Armus et al. 2007; Farrah et al. 2007a; Desai et al. 2007). The SHINING survey (Fischer et al. 2010; Sturm et al. 2011; Hailey-Dunsheath et al. 2012; González-Alfonso et al. 2013) obtained PACS spectroscopy for $19 / 43$ sources, so we observed, and present here, the remaining 24 objects. We also include Mrk 231 (Fischer et al. 2010) to give a final sample of 25 objects (Table 1). This sample is not flux limited, but does include nearly all ULIRGs at $z<0.27$ with $60 \mu \mathrm{m}$ fluxes between 1.7 Jy and $6 \mathrm{Jy}$, together with Mrk 231. The sample therefore gives an almost unbiased view of $z<0.3$ ULIRGs.

\subsection{Observations}

The PACS observations were performed between 2011 March 18 and 2012 April 8 (Operational Day 673-1060). The PACS integral-field spectrometer samples the spatial direction with 25 pixels and the spectral direction with 16 pixels. Each spectral pixel scans a distinct wavelength range by varying the grating angle. The combination of the 16 ranges constitutes the final spectrum. The resulting projection of the PACS array on the sky is a footprint of $5 \times 5$ spatial pixels (spaxels), corresponding to a $47^{\prime \prime} \times 47^{\prime \prime}$ field of view. The point-spread function (PSF) full width at half-maximum (FWHM) is $\approx 9^{\prime \prime} .5$ between $55 \mu \mathrm{m}$ and $110 \mu \mathrm{m}$, and increases to about $14^{\prime \prime}$ by $200 \mu \mathrm{m}$. A spaxel at the mean redshift of the sample is $\sim 3 \mathrm{kpc}$ in extent.

A single footprint observation was performed for each object as they are all smaller than the footprint size. The coordinates were chosen to place the optical centroids in the central spaxel. We observed the sample in the following lines: [O III $] 52 \mu \mathrm{m},[\mathrm{N}$ III $] 57 \mu \mathrm{m},\left[\mathrm{O}_{\mathrm{I}}\right] 63 \mu \mathrm{m},[\mathrm{N}$ II $] 122 \mu \mathrm{m}$, [O I] $145 \mu \mathrm{m}$, and [C II]158 $\mu \mathrm{m}$ (Table 2). Observations were performed in range spectroscopy mode. We used optical narrowline redshifts to set the central wavelengths of each range scan. For one object, IRAS $07598+6508$, the input coordinates were incorrect, placing the source near the edge of the PACS array, thus making the flux determination uncertain. We therefore substituted observations of this source from other programs. For [C II] we used the dataset 1342243534 (PI: Weedman), and for [N II] we used the dataset 1342231959 (PI: Veilleux).

We set the wavelength range of each range scan to accommodate uncertainties such as offsets between optical and far-IR line redshifts, and asymmetric or broadened lines. The chop/nod observation mode was used, in which the source is observed by alternating between the on-source position and a clean offsource position. Since the extent of the targets is always $<1^{\prime}$, the smallest throw $( \pm 1,5)$ was used to reduce the effect of fieldrotation between the two chop positions. Two nod positions were used in order to eliminate the telescope background emission.

The data reduction was performed in the Herschel Interactive Processing Environment (HIPE) version 8.0 (Ott 2010) using the default chop/nod pipeline script. The level one products (calibrated in flux and in wavelength, with bad pixel masks from HIPE) were then exported and processed by the PACSman tool (Lebouteiller et al. 2012).

\subsection{Line Measurements}

The best method to determine a line flux depends on the position and morphology of the line-emitting regions within the PACS footprint. If their combined spatial extent is significantly smaller than a single spaxel, are well centered on the central spaxel, and Herschel maintains an accurate pointing (the pointing accuracy of Herschel is $\sim 2$ ".5), then the best flux measurement is that of the central spaxel, scaled by an appropriate point-source correction. We call this method " $\mathcal{M} 1$." If these conditions are not satisfied, then $\mathcal{M} 1$ will give a lower limit on the flux.

We do not know a priori the morphologies of the far-IR line emitting regions, since high spatial resolution images of this 
Table 1

The Sample

\begin{tabular}{|c|c|c|c|c|c|c|c|}
\hline Galaxy & R.A. (J2000) & Decl. & Redshift & $L_{\mathrm{IR}}{ }^{\mathrm{a}}$ & Opt. Class & Stage $^{b}$ & SMBH Mass \\
\hline IRAS 00188-0856 & 002126.5 & -083926.3 & 0.128 & 12.39 & LINER & $\mathrm{V}$ & $\ldots$ \\
\hline IRAS 00397-1312 & 004215.5 & -125602.8 & 0.262 & 12.90 & H II & $\mathrm{V}$ & 0.11 \\
\hline IRAS 01003-2238 & 010250.0 & -222157.5 & 0.118 & 12.32 & H II & V & 0.25 \\
\hline Mrk 1014 & 015950.2 & +002340.6 & 0.163 & 12.62 & Sy1 & IIIb & 1.35 \\
\hline IRAS 03158+4227 & 031912.4 & +423828.0 & 0.134 & 12.63 & Sy 2 & IIIa & $\ldots$ \\
\hline IRAS $03521+0028$ & 035442.1 & +003703.4 & 0.152 & 12.52 & LINER & IIIb & $\ldots$ \\
\hline IRAS 06035-7102 & 060254.0 & -710310.2 & 0.079 & 12.22 & H II & IIIa & 0.09 \\
\hline IRAS 06206-6315 & 062101.2 & -631723.5 & 0.092 & 12.23 & Sy2 & $\mathrm{IIIb}$ & $\ldots$ \\
\hline IRAS 07598+6508 & 080433.1 & +645948.6 & 0.148 & 12.50 & Sy 1 & $\mathrm{IVb}$ & 1.48 \\
\hline IRAS 08311-2459 & 083320.6 & -250933.7 & 0.100 & 12.50 & Sy1 & IVa & $\ldots$ \\
\hline IRAS $10378+1109$ & 104029.2 & +105318.3 & 0.136 & 12.31 & LINER & $\mathrm{IVb}$ & 0.10 \\
\hline IRAS $11095-0238$ & 111203.4 & +020422.4 & 0.107 & 12.28 & LINER & IIIb & 0.35 \\
\hline IRAS $12071-0444$ & 120945.1 & -050113.9 & 0.128 & 12.41 & Sy2 & IIIb & 0.30 \\
\hline $3 \mathrm{C} 273$ & 122906.7 & +020308.6 & 0.158 & 12.83 & Sy1 & V & 24.2 \\
\hline Mrk 231 & 125614.2 & +56 5225.2 & 0.042 & 12.55 & Sy1 & $\mathrm{IVb}$ & 0.17 \\
\hline IRAS $13451+1232$ & 134733.3 & +121724.2 & 0.121 & 12.32 & Sy2 & IIIb & 0.53 \\
\hline Mrk 463 & 135602.9 & +182219.1 & 0.051 & 11.79 & Sy2 & IIIb & 0.65 \\
\hline IRAS $15462-0450$ & 154856.8 & -045933.6 & 0.100 & 12.24 & Sy1 & $\mathrm{IVb}$ & 0.69 \\
\hline IRAS 16090-0139 & 161140.5 & -014705.6 & 0.134 & 12.55 & LINER & IVa & $\ldots$ \\
\hline IRAS 19254-7245 & 193121.6 & -72 3922.0 & 0.063 & 12.09 & Sy2 & IIIb & 0.79 \\
\hline IRAS 20087-0308 & 201123.9 & -025950.7 & 0.106 & 12.42 & LINER & IVa & 1.94 \\
\hline IRAS $20100-4156$ & 201329.5 & -414734.9 & 0.130 & 12.67 & $\mathrm{H}_{\text {II }}$ & IIIb & $\ldots$ \\
\hline IRAS 20414-1651 & 204418.2 & -164016.2 & 0.087 & 12.22 & H II & $\mathrm{IVb}$ & 1.03 \\
\hline IRAS $23230-6926$ & 232603.6 & -691018.8 & 0.107 & 12.37 & LINER & IVa & 0.35 \\
\hline IRAS $23253-5415$ & 232806.1 & -535831.0 & 0.130 & 12.36 & $\mathrm{H}_{\text {II }}$ & IVa & $\ldots$ \\
\hline
\end{tabular}

Notes. Positions, redshifts and optical spectral classifications are taken from Saunders et al. 2000.

${ }^{a}$ Derived by combining the Spitzer IRS+MIPS data with all available far-IR photometry for each object, integrating under the IRS spectrum while spline-fitting to the longer wavelength data. See C. Borys et al. (in preparation) for details. The errors on the luminosities are approximately $20 \%$ in all cases.

${ }^{\mathrm{b}}$ Merger stage classification (Veilleux et al. 2002), updated using higher resolution imaging where appropriate (Rigopoulou et al. 1999; Meusinger et al. 2001; Farrah et al. 2001; Bushouse et al. 2002; Veilleux et al. 2006). IIIa: Premerger with separation >10 Kpc, IIIb: Premerger with separation $<10 \mathrm{Kpc}$, IVa: Diffuse merger (prominent tidal features, but only one nucleus), IVb: Compact merger, V: Undisturbed.

${ }^{c}$ SMBH mass, in units of $10^{8} M_{\odot}$ (Zheng et al. 2002; Dasyra et al. 2006; Greene \& Ho 2007; Zhang et al. 2008; Veilleux et al. 2009).

Table 2

Properties of the Lines Observed

\begin{tabular}{|c|c|c|c|c|c|c|c|}
\hline Line & $\begin{array}{l}\text { Wavelength } \\
\quad(\mu \mathrm{m})\end{array}$ & $\begin{array}{l}\mathrm{IP}_{1} \\
(\mathrm{eV})\end{array}$ & $\begin{array}{l}\mathrm{IP}_{2} \\
(\mathrm{eV})\end{array}$ & Configuration & $\begin{array}{c}n_{\mathrm{cr}, e} \\
\left(\mathrm{~cm}^{-3}\right)\end{array}$ & $\begin{array}{c}n_{\mathrm{cr}, H} \\
\left(\mathrm{~cm}^{-3}\right)\end{array}$ & $\begin{array}{l}T_{\mathrm{exc}} \\
(\mathrm{K})\end{array}$ \\
\hline [O III] & 51.815 & 35.12 & 54.93 & ${ }^{3} P_{2}-{ }^{3} P_{1}$ & $\simeq 3500$ & & 441 \\
\hline$[\mathrm{N}$ III] & 57.317 & 29.60 & 47.45 & ${ }^{2} P_{3 / 2}-{ }^{2} P_{1 / 2}$ & $\simeq 3000$ & & 251 \\
\hline$[\mathrm{OI}]$ & 63.184 & 2.00 & 13.62 & ${ }^{3} P_{1}-{ }^{3} P_{2}$ & $\simeq 2.8 \times 10^{5}$ & $\simeq 2.5 \times 10^{5}$ & 228 \\
\hline$[\mathrm{NII}]$ & 121.898 & 14.53 & 29.60 & ${ }^{3} P_{2}-{ }^{3} P_{1}$ & $\simeq 400$ & & 188 \\
\hline$\left[\mathrm{O}_{\mathrm{I}}\right]$ & 145.525 & $\ldots$ & 13.62 & ${ }^{3} P_{0}-{ }^{3} P_{1}$ & $\simeq 4 \times 10^{4}$ & $\simeq 5 \times 10^{4}$ & 327 \\
\hline [C II] & 157.741 & 11.26 & 24.38 & ${ }^{2} P_{3 / 2}-{ }^{2} P_{1 / 2}$ & $\simeq 40$ & $\simeq 2700$ & 91 \\
\hline
\end{tabular}

Note. Electron and hydrogen critical densities are given for $n=500 \mathrm{~cm}^{-3}$.

emission do not exist. Moreover, we cannot assume that the morphologies of these regions are traced reliably by emission at other wavelengths. We therefore are unable to straightforwardly distinguish between scenarios such as the source being centered and spatially extended, as opposed to the source being off-center and pointlike. Finally, we cannot assume that the morphologies of different lines in the same object are similar, since the line strengths are governed by different excitation temperatures and critical densities.

Given these caveats, there are five further methods to determine a line flux.

1. $\mathcal{M} 2$. Fit line profiles to the central $3 \times 3$ spaxels individually, sum the resulting fluxes, and apply a point-source correction (of the order of 15\% or less of the total flux) that accounts for the additional area sampling of the PSF. This method is suitable if the source is spatially extended or shifted by at most a significant fraction of a spaxel. The point-source correction is wavelength-dependent, but since our range scans span wavelength ranges of the order of $1 \mu \mathrm{m}$, it is equivalent to either calculate the flux and then apply the correction, or apply the correction to the spectrum and then calculate the flux.

2. M3. Co-add the spectra of the central $3 \times 3$ spaxels, apply a point-source correction, and fit a line profile to the combined profile. This method is identical to $\mathcal{M} 2$, except for additional uncertainties from combining spectra with 
differently shaped continua. We mention this method for completeness but do not use it.

3. M4. Fit line profiles to those spaxels with a line detection, then sum the resulting fluxes. This is appropriate if the source is extended in any fashion, but suffers from uncertainties due to imperfect knowledge of the source morphology.

4. M5. Fit line profiles to every spaxel in the PACS array and sum them. The point-source correction for this method is negligible. This method will capture all of the line flux, but will overestimate uncertainties unless the source is both bright and extended across at least most of the PACS field of view.

5. M0. Fit a PSF, as a function of position and intensity, across the whole PACS array and adopt the best fit. This is a photometric equivalent of "optimal" extraction as described in Lebouteiller et al. (2010).

To choose the method for the lines in each source, we proceed as follows. First, we determine all six measurements for each line. If the emission is consistent with a well-centered point source, then the fluxes from all six methods will agree with each other, with larger errors for the methods that include more spaxels. We found this to be the case in the majority of the sample. For these, we adopted $\mathcal{M} 1$. For the others, the measurements from $\mathcal{M} 2$ through $\mathcal{M} 6$ were higher than $\mathcal{M} 1$, but were consistent with each other. This indicates that the line emission is mostly confined to the central $3 \times 3$ spaxels. We therefore discarded $\mathcal{M} 5$. To avoid the uncertainties involved in using a simulated PSF, in most cases we then used the measurements from $\mathcal{M} 2$ rather than $\mathcal{M} 4$ or $\mathcal{M} 6$, even though the $\mathcal{M} 2$ errors are larger. In most cases, for each object, the same measurement method was used for all lines. However, in a few cases, $[\mathrm{C}$ II $]$ is extended while the other lines are consistent with point sources.

For one object, IRAS 00397-1312, [C II] is redshifted such that it lies in a part of the PACS wavelength range that suffers from significant flux leakage. In this wavelength range, which spans approximately $190 \mu \mathrm{m}$ to $210 \mu \mathrm{m}$, there is superimposed emission from the second order, at $95-110 \mu \mathrm{m}$. Since [C II] in IRAS 00397-1312 was observed with SPIRE, we substitute the SPIRE-FTS measurement for this line.

Except for [C II] in IRAS 00397-1312, line fits were performed with PACSman for each spaxel at each raster position, using all of the points in the data cloud. Errors were estimated from the dispersion of the cloud in each wavelength bin. The fitting function was a Gaussian profile, adjusted simultaneously with a polynomial continuum of degrees one to three. The instrument spectral resolution ranges from $\sim 55 \mathrm{~km} \mathrm{~s}^{-1}$ to $\sim 320 \mathrm{~km} \mathrm{~s}^{-1}$, depending on the band, order, and wavelength. The intrinsic line broadening FWHM was determined by calculating the quadratic difference between the observed FWHM and the instrumental FWHM, assuming Gaussian profiles.

\section{RESULTS AND ANALYSIS}

We tabulate the far-infrared line properties in Table 3, and present their profiles in Figures 1-3. In the following analysis, we frequently compare the far-IR line properties to those of the mid-IR fine-structure lines (Farrah et al. 2007a), the $6.2 \mu \mathrm{m}$ and $11.2 \mu \mathrm{m}$ polycyclic aromatic hydrocarbon (PAH) features, and the $9.7 \mu \mathrm{m}$ silicate feature (Table 4). The PAH and silicate feature data were measured from spectra taken from the CASSIS v4 database (Lebouteiller et al. 2011). The PAH luminosities were measured by integrating over 5.9-6.6 $\mu \mathrm{m}$ and $10.8-11.8 \mu \mathrm{m}$ in the continuum subtracted spectra, respectively (Spoon et al. 2007). For the silicate feature, we define its strength, $S_{\mathrm{Sil}}$, as

$$
S_{\mathrm{Sil}}=\ln \left[\frac{f_{\mathrm{obs}}}{f_{\text {cont }}}\right],
$$

where $f_{\text {obs }}$ is the observed flux at rest-frame $9.7 \mu \mathrm{m}$ and $f_{\text {cont }}$ is the continuum flux at rest-frame $9.7 \mu \mathrm{m}$ in the absence of silicate absorption, inferred from a spline fit to the continuum at 5-7 $\mu \mathrm{m}$ and 14-14.5 $\mu \mathrm{m}$ (Spoon et al. 2007; Levenson et al. 2007). A positive value corresponds to silicates in absorption.

\subsection{Line Properties}

\subsubsection{Profile Shapes}

In most cases, the lines are reproducible by single Gaussians with widths between $250 \mathrm{~km} \mathrm{~s}^{-1}$ and $600 \mathrm{~km} \mathrm{~s}^{-1}$. We do not see greater widths in the higher ionization lines. We also do not see strong asymmetries or systemic offsets in the velocity (compared to the optical redshift) of any line. In a few cases, the line profiles are not reproducible with single symmetric profiles. The [O I]63 lines in two objects, IRAS 06206-6315 and IRAS 20414-1651, are consistent with significant selfabsorption (see also Graciá-Carpio et al. 2011). There is weaker evidence for [OI]63 self absorption in IRAS 00188-0856, IRAS 11095-0238, and IRAS 19254-7245. Self-absorption in [O I]63 can occur when cool oxygen in foreground clouds reduces the [O I]63 flux (Poglitsch et al. 1996; Fischer et al. 1999; Vastel et al. 2002; Luhman et al. 2003; Okada et al. 2003). The [C II] profiles in IRAS 20087-0308, IRAS 20414-4651, and IRAS 23253-5415 may show subtle asymmetries. Finally, in four cases (IRAS 06035-7102, Mrk 463, IRAS 11095-0238, and IRAS 20100-4156), [C II] may show an additional, broad emission component with widths between $600 \mathrm{~km} \mathrm{~s}^{-1}$ and $1200 \mathrm{~km} \mathrm{~s}^{-1}$. For consistency with the other lines, we do not include the broad component in the line fluxes in Table 3, but instead discuss it in a future paper.

We examine the distribution of line widths in Figure 4, using only the narrow components in those cases where an additional broad one exists. The ranges in width for all six lines are consistent with each other. We see no dependence of the ranges in width on optical class. For individual objects, however, there are sometimes substantial differences between individual line widths. In Mrk 1014, for example, the [C II] and $[\mathrm{NII}]$ line widths differ by nearly a factor of two. We speculate that these differences are due to one or more of the following: (1) differences in the critical densities of the lines, (2) an individually unresolved broad component in one line is brighter than the equivalent component in the other line, and (3) in the case of [C II] and [N II], the [N II] emission arises mostly from $\mathrm{H}$ II regions, while at least some of the $[\mathrm{C}$ II $]$ emission arises from photodissociation regions (PDRs) or the diffuse interstellar medium (ISM). It is, however, also possible that the [N II] profile is affected by the $4_{32}-4_{23}$ transition of o- $\mathrm{H}_{2} \mathrm{O}$ at $121.72 \mu \mathrm{m}$ (Fischer et al. 2010; González-Alfonso et al. 2010). This is discussed in Spoon et al. (2013).

\subsubsection{Luminosities}

We plot the luminosities of selected individual lines against each other in Figure 5. The line luminosities range from just under $10^{7} L_{\odot}$ to $\sim 3 \times 10^{9} L_{\odot}$. The [C II] or [O I] 63 lines are 
Far-infrared Line Fluxes

\begin{tabular}{|c|c|c|c|c|c|c|c|c|c|c|c|c|c|c|c|c|c|c|}
\hline Galaxy & & III] & & & $\mathrm{N}_{\text {IIII }}$ & & {$[\mathrm{O}$} & I]63 & & & $\left.\mathrm{N}_{\text {II }}\right]$ & & & I] 145 & & & III] & \\
\hline IRAS 00188-0856 & $0.64 \pm 0.80$ & $350 \pm 349$ & (1) & $<0.52$ & $350 \pm 159$ & (1) & $3.54 \pm 0.71^{\mathrm{a}}$ & $225 \pm 36$ & (1) & $0.85 \pm 0.51$ & $296 \pm 38$ & (2) & $89 \pm 0.39$ & $350 \pm 53$ & (2) & $.85 \pm 1.02$ & $279 \pm 13$ & (2) \\
\hline IRAS $00397-1312$ & $3.05 \pm 0.43$ & $259 \pm 40$ & (1) & $1.17 \pm 0.31$ & $372 \pm 90$ & (1) & $4.99 \pm 0.49$ & $328 \pm 30$ & (1) & $0.35 \pm 0.09$ & $333 \pm 85$ & (1) & $0.22 \pm 0.09$ & $250 \pm 94$ & (1) & $1.61 \pm 0.25^{\mathrm{b}}$ & $427 \pm 61$ & \\
\hline IRAS 01003-2238 & $3.20 \pm 1.00$ & $400 \pm 99$ & (1) & $0.72 \pm 0.29$ & $150 \pm 48$ & (1) & $8.65 \pm 1.61$ & $197 \pm 34$ & (1) & $0.08 \pm 0.16^{\mathrm{c}}$ & $400 \pm 639$ & (1) & $0.49 \pm 0.12$ & $165 \pm 39$ & (1) & $5.36 \pm 1.23^{\mathrm{d}}$ & $150 \pm 7$ & (2) \\
\hline Mrk 1014 & $4.43 \pm 4.15$ & $270 \pm 59$ & (2) & $3.83 \pm 4.33$ & $250 \pm 253$ & (2) & $6.89 \pm 4.39$ & $350 \pm 41$ & (2) & $1.21 \pm 0.55$ & $507 \pm 50$ & (2) & $0.88 \pm 0.72$ & $358 \pm 69$ & (2) & $4.33 \pm 1.07$ & $316 \pm 17$ & (2) \\
\hline IRAS 03158+4227 & $5.38 \pm 4.29$ & $300 \pm 178$ & (2) & $0.78 \pm 3.20$ & $150 \pm 87$ & (2) & $9.69 \pm 6.20$ & $155 \pm 47$ & (2) & $0.83 \pm 0.58$ & $150 \pm 45$ & (2) & $1.30 \pm 0.69$ & $214 \pm 48$ & (2) & $5.63 \pm 0.11$ & $243 \pm 4$ & (2) \\
\hline IRAS $03521+0028$ & $4.74 \pm 4.60$ & $350 \pm 232$ & (2) & $3.17 \pm 2.87$ & $350 \pm 169$ & (2) & $4.62 \pm 2.94$ & $500 \pm 145$ & (2) & $0.64 \pm 0.43$ & $351 \pm 102$ & (2) & $0.68 \pm 0.70$ & $350 \pm 114$ & (2) & $3.75 \pm 1.08$ & $442 \pm 27$ & (2) \\
\hline IRAS $06035-7102$ & $9.52 \pm 10.53$ & $215 \pm 105$ & (2) & $3.05 \pm 4.42$ & $400 \pm 134$ & (2) & $24.89 \pm 7.66$ & $322 \pm 16$ & (2) & $2.52 \pm 0.60$ & $373 \pm 21$ & (2) & $2.29 \pm 0.70$ & $242 \pm 16$ & (2) & $30.58 \pm 2.04^{\mathrm{d}}$ & $277 \pm 2$ & (4) \\
\hline IRAS 06206-6315 & $4.56 \pm 5.54$ & $350 \pm 247$ & (2) & $3.99 \pm 3.38$ & $350 \pm 146$ & (2) & $10.27 \pm 8.77^{a}$ & $350 \pm 170$ & (2) & $1.47 \pm 0.53$ & $418 \pm 45$ & (2) & $1.26 \pm 0.64$ & $450 \pm 66$ & (2) & $9.25 \pm 1.19$ & $369 \pm 10$ & (2) \\
\hline IRAS 07598+6508 & $0.34 \pm 2.20$ & $150 \pm 127$ & (2) & $1.33 \pm 1.87$ & $150 \pm 237$ & (2) & $1.32 \pm 1.25$ & $153 \pm 101$ & (0) & $0.67 \pm 0.31$ & $282 \pm 60$ & (2) & $0.39 \pm 0.39$ & $184 \pm 74$ & (1) & $2.10 \pm 0.38$ & $197 \pm 35$ & (1) \\
\hline IRAS 08311-2459 & $14.42 \pm 1.54$ & $379 \pm 32$ & (1) & $5.20 \pm 0.50$ & $263 \pm 20$ & (1) & $29.16 \pm 1.08$ & $6 \pm 9$ & (1) & $3.03 \pm$ & $334 \pm 14$ & (1) & $2.16 \pm 0.15$ & $348 \pm 20$ & (1) & $24.32 \pm 0.35$ & $272 \pm 3$ & (1) \\
\hline IRAS $10378+1109$ & $1.09 \pm 0.80$ & $351 \pm 211$ & (1) & $0.99 \pm 0.46$ & $350 \pm 131$ & (1) & $5.46 \pm 1.17$ & $466 \pm 83$ & (1) & $0.71 \pm 0.12$ & $439 \pm 64$ & (1) & $0.55 \pm 0.14$ & $475 \pm 101$ & (1) & $3.53 \pm 0.20$ & $409 \pm 20$ & (1) \\
\hline IRAS $11095-0238$ & $4.45 \pm 1.25$ & $323 \pm 73$ & (1) & $1.16 \pm 0.82$ & $356 \pm 202$ & (1) & $8.58 \pm 1.28^{\mathrm{a}}$ & $343 \pm 41$ & (1) & $0.63 \pm 0.09$ & $375 \pm 46$ & (1) & $1.40 \pm 0.54$ & $358 \pm 43$ & (2) & $\pm 1.18^{\mathrm{d}}$ & $296 \pm 9$ & (2) \\
\hline IRAS $12071-0444$ & $2.01 \pm 0.71$ & $300 \pm 85$ & (1) & $0.55 \pm 0.36$ & $225 \pm 118$ & (1) & $6.43 \pm 0.78$ & $225 \pm 22$ & (1) & $0.48 \pm 0.11$ & $225 \pm 42$ & (1) & $0.81 \pm 0.14$ & $295 \pm 43$ & (1) & $8 \pm 0.90^{\mathrm{d}}$ & $250 \pm 6$ & (2) \\
\hline $3 \mathrm{C} 273$ & $2.84 \pm 0.83$ & $461 \pm 111$ & (1) & $<0.40$ & $300 \pm 400$ & (1) & 77 & $418 \pm 92$ & (1) & 0.31 & $500 \pm 134$ & (1) & $0.17 \pm 0.13$ & $500 \pm 321$ & (1) & $1.47=$ & $465 \pm 66$ & (1) \\
\hline $\operatorname{Mrk} 231^{\mathrm{e}}$ & & & & $2.80 \pm 0.60$ & 177 & & 36.00 & 218 & & 4.10 & 266 & & $3.20 \pm 0.40$ & 208 & & 38.30 & 247 & \\
\hline RAS $13451+1232$ & $<0.38$ & $300 \pm 101$ & (1) & $1.30 \pm 0.59$ & $350 \pm 154$ & (1) & $9.01 \pm 1.10$ & $467 \pm 46$ & (1) & $0.41 \pm 0.07$ & $300 \pm 42$ & (1) & $0.46 \pm 0.10$ & $300 \pm 53$ & (1) & .21 & $465 \pm 18$ & (1) \\
\hline Irk 463 & $28.73 \pm 13.84$ & $272 \pm 23$ & (2) & $566+$ & $369 \pm 70$ & (2) & 28. & 15 & (2) & 63 & 42 & (2) & .59 & 25 & (2) & $21.33 \pm 0.88^{\mathrm{d}}$ & 25 & (2) \\
\hline IRAS $15462-0450$ & $8.45 \pm 5.27$ & $125 \pm 17$ & (2) & $3.30 \pm 1.94$ & $350 \pm 76$ & (2) & $14.41=$ & $258=$ & (2) & 0.79 & $125 \pm 14$ & (2) & $1.28 \pm 0.58$ & $252 \pm 35$ & (2) & 7.40 & $163 \pm 4$ & (4) \\
\hline IRAS $16090-0139$ & $10.11 \pm 5.21$ & $500 \pm 104$ & (2) & $3.33 \pm 3.15$ & $370 \pm 156$ & (2) & $13.13=$ & $357 \pm 40$ & (2) & $0.97 \pm$ & $464 \pm 50$ & (2) & $1.79 \pm 0.66$ & $437 \pm 48$ & (2) & 9.54 & $352 \pm 8$ & (2) \\
\hline IRAS 19 & $8.86 \pm 15.21$ & $475 \pm 211$ & (2) & $4.18 \pm 6.19$ & $475 \pm 642$ & (2) & $18.24 \pm 12.46^{\mathrm{a}}$ & $475 \pm 89$ & (2) & 3.85 & $600 \pm 38$ & (2) & 2.30 & $520 \pm 59$ & (2) & 31.37 & $=12$ & (2) \\
\hline IRAS 20087-0308 & $2.92 \pm 11.26$ & $475 \pm 315$ & (2) & $2.93 \pm 5.32$ & $550 \pm 362$ & (2) & $6.02 \pm 1.14$ & $511 \pm 72$ & (1) & $2.35 \pm$ & $550 \pm 18$ & (1) & $1.81 \pm 0.73$ & $489 \pm 55$ & (2) & $17.14 \pm 0.18$ & $550 \pm 5$ & (2) \\
\hline IRAS $20100-4156$ & $4.90 \pm 0.81$ & $202 \pm 27$ & (1) & $1.65 \pm 0.43$ & $300 \pm 64$ & (1) & $8.02 \pm 0$ & $235 \pm 19$ & (1) & $0.92 \pm 0.08$ & & (1) & $0.96 \pm 0.10$ & & (1) & 9.65 & $247 \pm 4$ & (1) \\
\hline IRAS 20414-1651 & $1.24 \pm 1.14$ & $550 \pm 389$ & (1) & $1.24 \pm 0.76$ & $475 \pm 223$ & (1) & $0.24 \pm 1.64^{\mathrm{a}}$ & $550 \pm 895$ & (1) & $1.21 \pm$ & $550 \pm 42$ & (1) & $1.19 \pm 0.14$ & $550 \pm 50$ & (1) & $9.13 \pm 0.46$ & $475 \pm 12$ & (2) \\
\hline IRAS $23230-6926$ & $4.02 \pm 0.91$ & $250 \pm 44$ & (1) & $1.29 \pm 0.76$ & $375 \pm 173$ & (1) & 5.72 & $288 \pm 46$ & (1) & & $375 \pm 43$ & (1) & $1.32 \pm 0.15$ & $279 \pm 27$ & (1) & 9.99 & $307 \pm 5$ & (1) \\
\hline IRAS $23253-5415$ & $<0.76$ & $375 \pm 648$ & (1) & $0.80 \pm 0.52$ & $375 \pm 194$ & (1) & $9.47 \pm 5.81$ & $503 \pm 88$ & (2) & $0.83 \pm 0.12$ & $411 \pm 49$ & (1) & $0.53 \pm 0.12$ & $375 \pm 69$ & (1) & $12.57 \pm 1.08^{\mathrm{d}}$ & $473 \pm 8$ & (2) \\
\hline
\end{tabular}

Notes. For each line, first the flux is given in units of $\times 10^{-21} \mathrm{~W} \mathrm{~cm}^{-2}$, then the line width in $\mathrm{km} \mathrm{s}^{-1}$, then the method used to measure the flux is in brackets (Section 2.3).

${ }^{a}$ May be self-absorbed, see Section 3.1.1.

${ }^{\mathrm{b}}$ SPIRE-FTS flux, see Section 2.3.

${ }^{c}$ May be unusually low, see Section 3.1.2

d May show asymmetry or an additional broad component, see Section 3.1.1.

e Measurements taken from Fischer et al. (2010). 

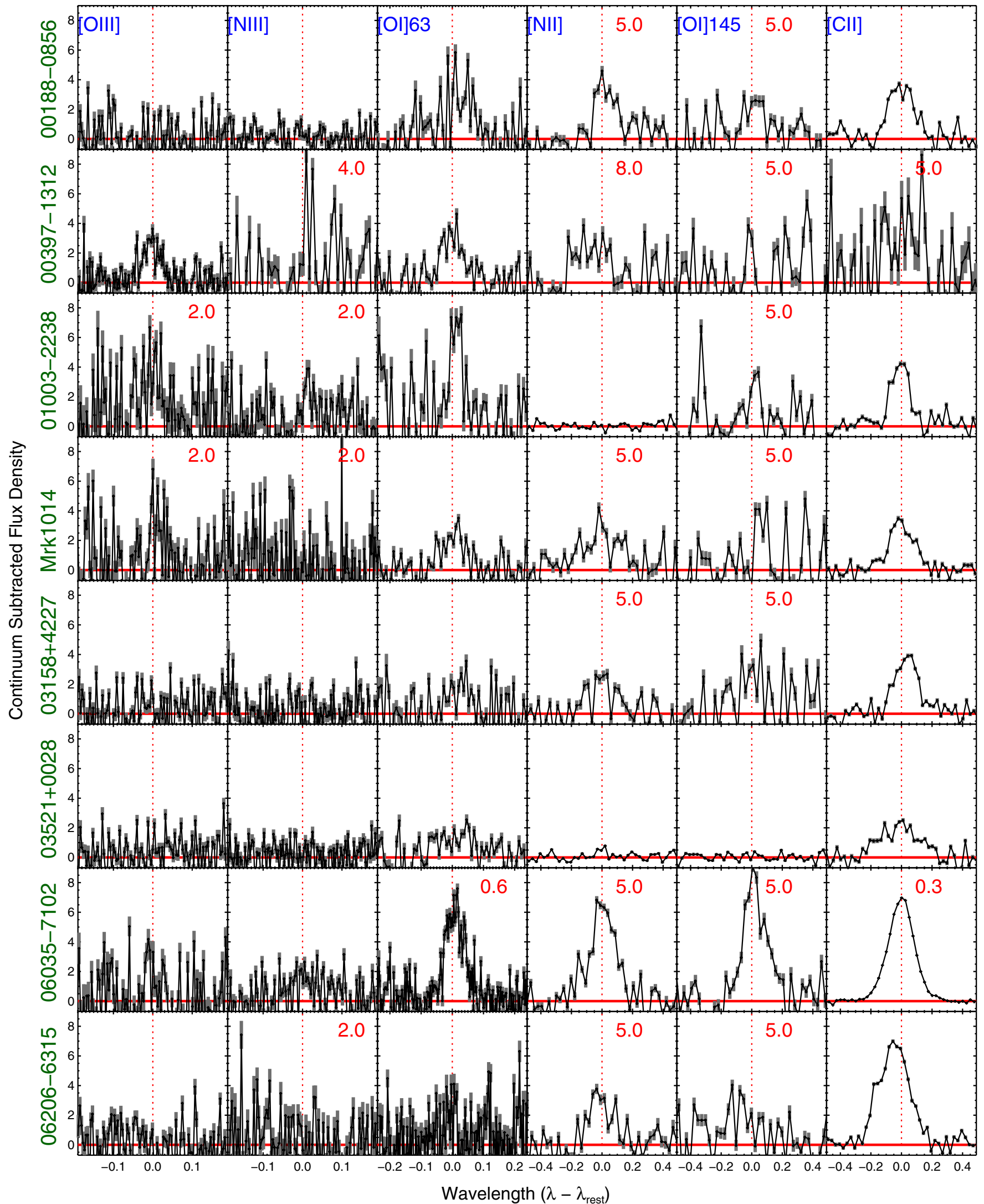

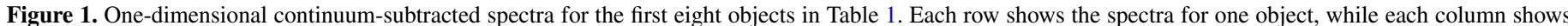

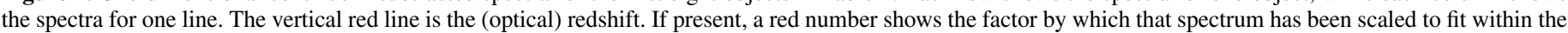
$y$ axis range. We plot the rebinned spectra rather than the full data cloud and show data only from the central spaxel.

(A color version of this figure is available in the online journal.) 

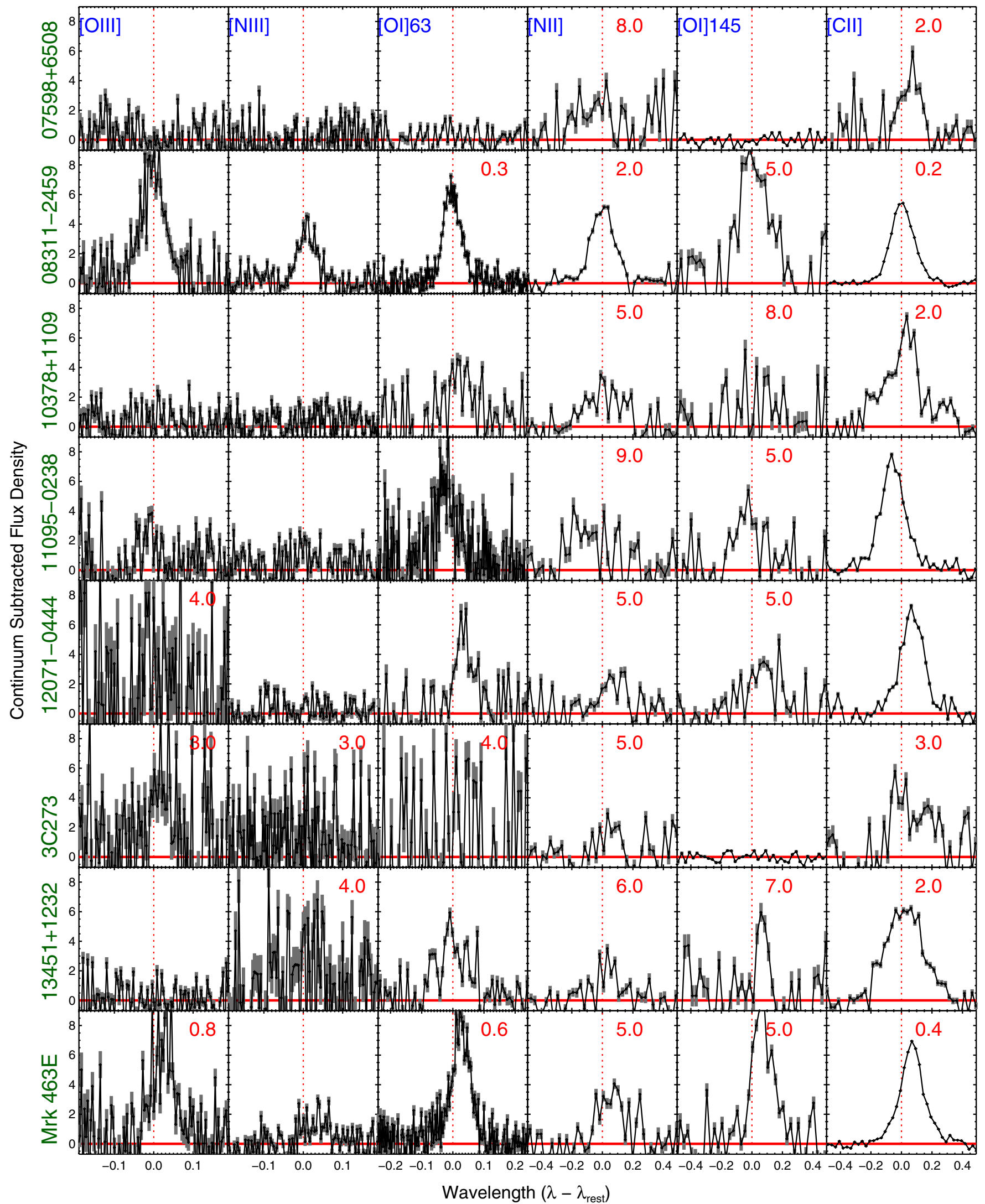

Figure 2. One-dimensional spectra of each line for the second eight objects in Table 1, excluding Mrk 231 (see Fischer et al. 2010). The labeling of the plot is the same as in Figure 1.

(A color version of this figure is available in the online journal.) 


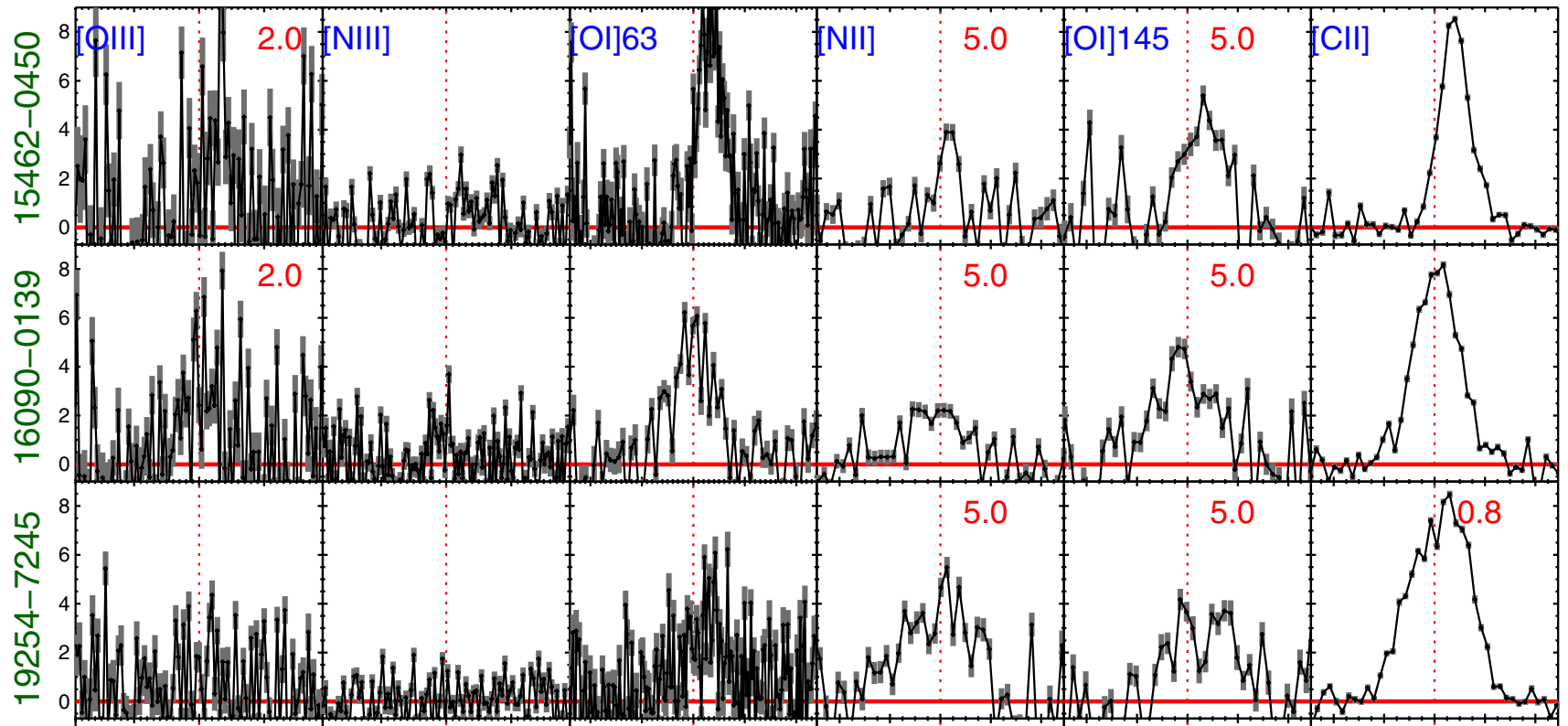

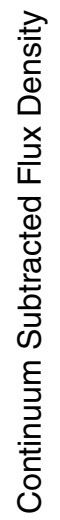
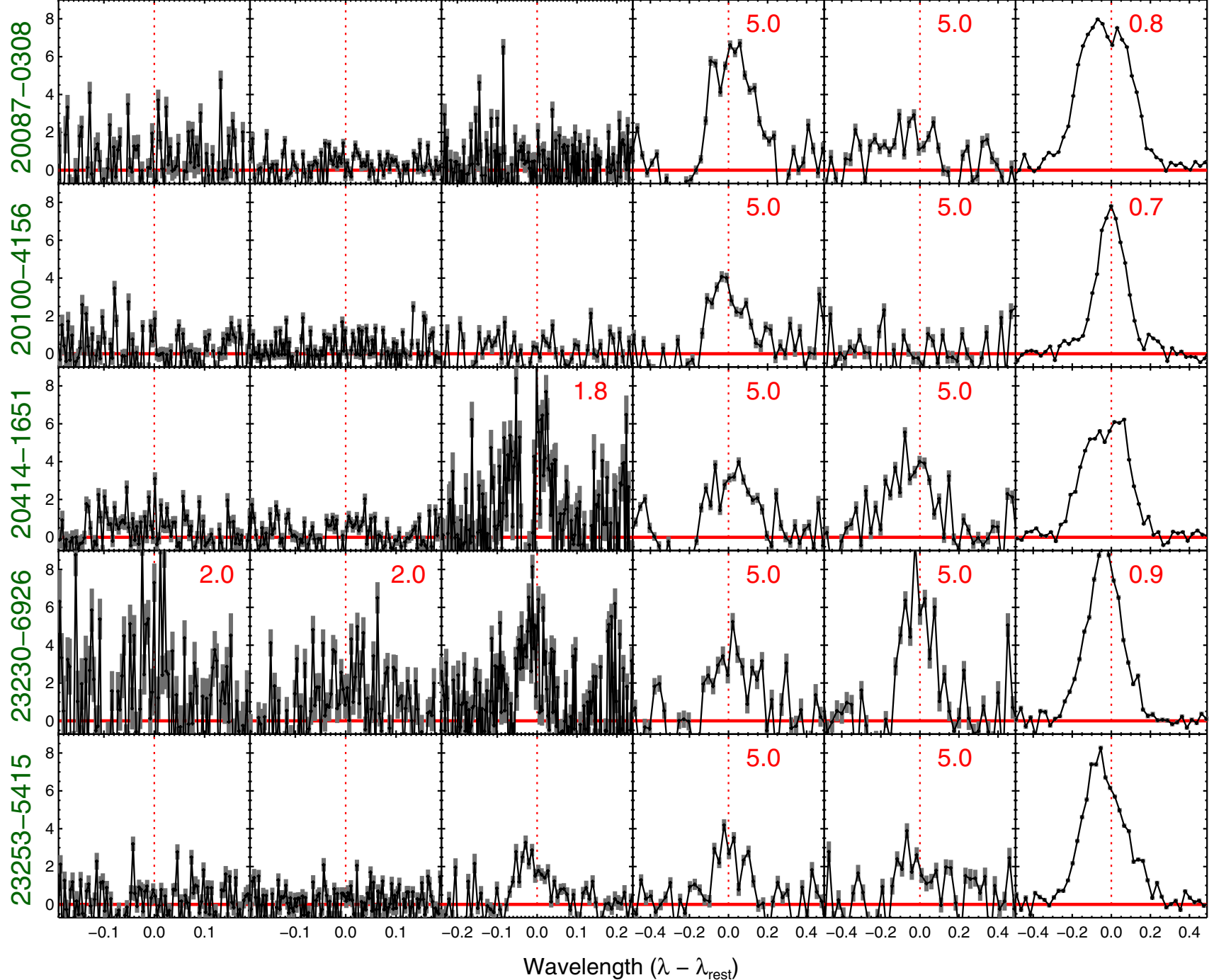

Figure 3. One-dimensional spectra of each line for the last eight objects in Table 1. The labeling of the plot is the same as in Figure 1.

(A color version of this figure is available in the online journal.) 
Table 4

Mid-IR Properties and Far-IR Derived Star Formation Rates

\begin{tabular}{|c|c|c|c|c|c|c|c|}
\hline \multirow[t]{2}{*}{ Galaxy } & \multicolumn{2}{|c|}{ PAH 6.2} & \multicolumn{2}{|c|}{ PAH 11.2} & \multirow[t]{2}{*}{$S_{\mathrm{Sil}}$} & \multirow{2}{*}{$\begin{array}{c}\left\langle\mathrm{SFR}_{\text {Line }}\right\rangle \\
\left(M_{\odot} \mathrm{yr}^{-1}\right)\end{array}$} & \multirow{2}{*}{$\begin{array}{l}\left\langle\mathrm{SFR}_{\mathrm{Cont}}\right\rangle \\
\left(M_{\odot} \mathrm{yr}^{-1}\right)\end{array}$} \\
\hline & Flux & EW & Flux & EW & & & \\
\hline IRAS 00188-0856 & $1.89 \pm 0.28$ & $0.065 \pm 0.009$ & $1.45 \pm 0.10$ & $0.39 \pm 0.01$ & $2.62 \pm 0.01$ & $98 \pm 25$ & $239 \pm 67$ \\
\hline IRAS 00397-1312 & $1.81 \pm 0.28$ & $0.026 \pm 0.004$ & $0.80 \pm 0.22$ & $0.19 \pm 0.06$ & $2.94 \pm 0.01$ & $655 \pm 207$ & $979 \pm 195$ \\
\hline IRAS 01003-2238 & $1.02 \pm 0.07$ & $0.039 \pm 0.002$ & $0.65 \pm 0.14$ & $0.02 \pm 0.01$ & $0.77 \pm 0.08$ & $129 \pm 34$ & $97 \pm 14$ \\
\hline Mrk 1014 & $1.91 \pm 0.22$ & $0.079 \pm 0.009$ & $1.75 \pm 0.12$ & $0.07 \pm 0.01$ & $-0.21 \pm 0.02$ & $183 \pm 60$ & $\ldots$ \\
\hline IRAS $03158+4227$ & $1.30 \pm 0.11$ & $0.059 \pm 0.005$ & $1.19 \pm 0.12$ & $0.43 \pm 0.09$ & $3.13 \pm 0.01$ & $157 \pm 17$ & $270 \pm 22$ \\
\hline IRAS $03521+0028$ & $1.05 \pm 0.07$ & $0.355 \pm 0.035$ & $0.94 \pm 0.07$ & $0.50 \pm 0.01$ & $1.29 \pm 0.13$ & $137 \pm 28$ & $252 \pm 80$ \\
\hline IRAS 06035-7102 & $4.42 \pm 0.47$ & $0.087 \pm 0.009$ & $3.88 \pm 0.27$ & $0.28 \pm 0.01$ & $1.51 \pm 0.01$ & $219 \pm 23$ & $138 \pm 24$ \\
\hline IRAS 06206-6315 & $2.80 \pm 0.40$ & $0.183 \pm 0.026$ & $1.96 \pm 0.14$ & $0.35 \pm 0.01$ & $1.57 \pm 0.16$ & $117 \pm 22$ & $162 \pm 24$ \\
\hline IRAS 07598+6508 & $8.25 \pm 0.27$ & $0.006 \pm 0.002$ & $0.78 \pm 0.24$ & $0.01 \pm 0.005$ & $-0.13 \pm 0.01$ & $75 \pm 16$ & $\ldots$ \\
\hline IRAS 08311-2459 & $6.38 \pm 0.40$ & $0.139 \pm 0.006$ & $8.52 \pm 0.30$ & $0.06 \pm 0.01$ & $0.49 \pm 0.05$ & $436 \pm 31$ & $190 \pm 21$ \\
\hline IRAS $10378+1109$ & $9.16 \pm 0.18$ & $0.090 \pm 0.017$ & $0.70 \pm 0.05$ & $0.44 \pm 0.03$ & $2.13 \pm 0.01$ & $153 \pm 13$ & $149 \pm 13$ \\
\hline IRAS $11095-0238$ & $1.20 \pm 0.50$ & $0.037 \pm 0.015$ & $1.10 \pm 0.12$ & $0.40 \pm 0.07$ & $3.28 \pm 0.01$ & $131 \pm 21$ & $118 \pm 12$ \\
\hline IRAS 12071-0444 & $1.66 \pm 0.13$ & $0.087 \pm 0.006$ & $1.20 \pm 0.01$ & $0.08 \pm 0.01$ & $1.37 \pm 0.01$ & $171 \pm 50$ & $143 \pm 16$ \\
\hline $3 \mathrm{C} 273$ & $<1.16$ & $<0.007$ & $<0.32$ & $<0.004$ & $-0.11 \pm 0.01$ & $153 \pm 59$ & $56 \pm 19$ \\
\hline Mrk 231 & $6.48 \pm 1.53$ & $0.009 \pm 0.002$ & $9.02 \pm 0.63$ & $0.03 \pm 0.005$ & $0.65 \pm 0.07$ & $65 \pm 17$ & $\ldots$ \\
\hline IRAS 13451+1232 & $<5.10$ & $<0.02$ & $<0.6$ & $<0.02$ & $0.26 \pm 0.03$ & $131 \pm 61$ & $103 \pm 11$ \\
\hline Mrk 463 & $<1.04$ & $<0.01$ & $<4.25$ & $<0.04$ & $0.41 \pm 0.04$ & $90 \pm 27$ & $\ldots$ \\
\hline IRAS $15462-0450$ & $1.79 \pm 0.13$ & $0.061 \pm 0.002$ & $1.36 \pm 0.11$ & $0.07 \pm 0.01$ & $0.38 \pm 0.04$ & $190 \pm 33$ & $\cdots$ \\
\hline IRAS 16090-0139 & $2.09 \pm 0.19$ & $0.070 \pm 0.006$ & $1.74 \pm 0.12$ & $0.44 \pm 0.01$ & $2.52 \pm 0.01$ & $241 \pm 16$ & $289 \pm 29$ \\
\hline IRAS $19254-7245$ & $6.66 \pm 0.93$ & $0.066 \pm 0.009$ & $4.59 \pm 0.32$ & $0.12 \pm 0.01$ & $1.33 \pm 0.01$ & $165 \pm 20$ & $90 \pm 20$ \\
\hline IRAS 20087-0308 & $5.37 \pm 0.39$ & $0.366 \pm 0.026$ & $2.85 \pm 0.20$ & $0.70 \pm 0.01$ & $1.80 \pm 0.18$ & $216 \pm 118$ & $319 \pm 13$ \\
\hline IRAS 20100-4156 & $2.94 \pm 0.65$ & $0.088 \pm 0.019$ & $2.12 \pm 0.15$ & $0.61 \pm 0.05$ & $2.66 \pm 0.01$ & $254 \pm 35$ & $496 \pm 149$ \\
\hline IRAS 20414-1651 & $2.91 \pm 0.20$ & $0.570 \pm 0.014$ & $1.59 \pm 0.11$ & $0.58 \pm 0.01$ & $1.61 \pm 0.16$ & $106 \pm 12$ & $94 \pm 38$ \\
\hline IRAS 23230-6926 & $3.27 \pm 0.42$ & $0.323 \pm 0.041$ & $1.79 \pm 0.12$ & $0.54 \pm 0.01$ & $2.13 \pm 0.01$ & $161 \pm 64$ & $139 \pm 16$ \\
\hline IRAS $23253-5415$ & $1.30 \pm 0.26$ & $0.234 \pm 0.068$ & $1.25 \pm 0.09$ & $0.41 \pm 0.01$ & $1.46 \pm 0.15$ & $203 \pm 82$ & $194 \pm 59$ \\
\hline
\end{tabular}

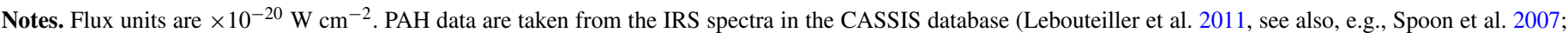

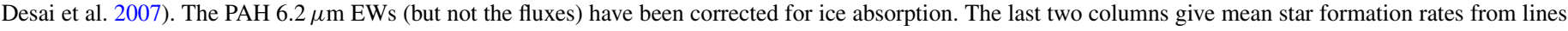
and continua detected at $>3 \sigma$, using Equations (6a)-(6f) and (8a)-(8f).

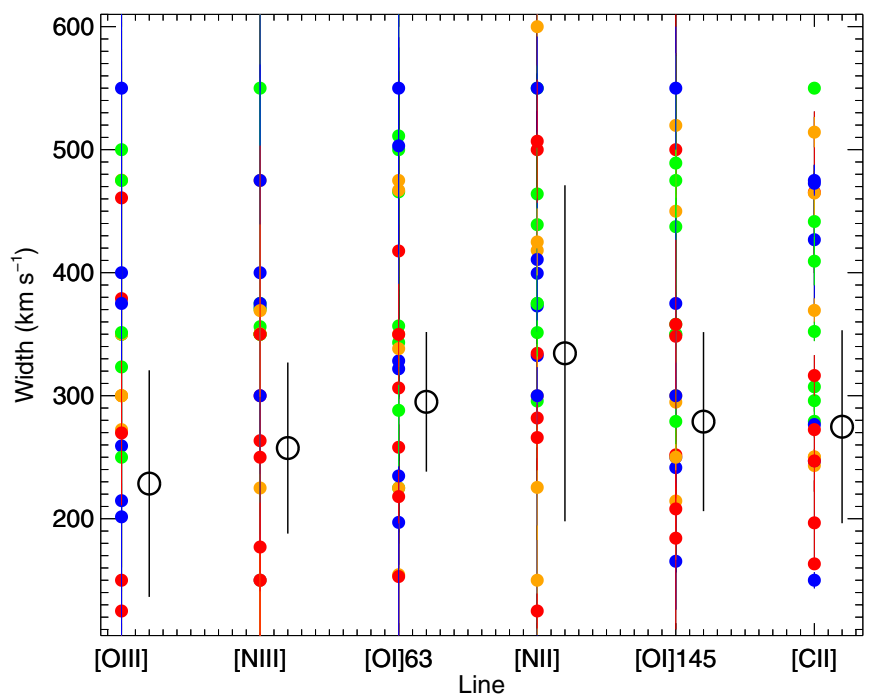

Figure 4. Distribution of line widths for each line, color coded by optical class (blue: H II, green: LINER, orange: Sy2, red: Sy1). See Section 3.1.1 for discussion. Objects with errors on their widths exceeding $30 \%$ are not plotted. The open black circles show the (weighted) mean and error for each line. The line widths are corrected for instrumental broadening.

(A color version of this figure is available in the online journal.)

usually the most luminous lines, though the [O III] line may be the second-most luminous in 3C273 and IRAS 00397-1312. The two least-luminous lines in most cases are [N $\mathrm{NI}]$ and $[\mathrm{OI}] 145$. In some cases, $[\mathrm{N}$ III] is consistent with being the least luminous, and in a few cases (e.g., IRAS 07598+6508 IRAS 23253-5415) [O III] is consistent with being the least luminous. The range in luminosities decreases approximately with increasing wavelength; the [O III] and [N III] line luminosities span a factor of $\sim 50$, whereas the [C II] luminosity spans only a factor of five, which is less than the range in $L_{\mathrm{IR}}$ of the sample.

To determine if the line luminosities correlate with each other, we fit the relation in Equation (2) (see Section 3.2.1 for methodology). We find positive correlations in all cases, with $\beta$ values mostly between 0.5 and 1.2. In no case, however, is the correlation particularly strong (in terms of the $\mathrm{S} / \mathrm{N}$ on $\beta$ ). We find that among these six lines, the luminosity of one cannot be used to predict the luminosity of another to better than $\sim 0.2$ dex. If we instead plot line luminosities normalized by $L_{\mathrm{IR}}$ (Figure 6), then the correlations do not change significantly; they do not depend on optical spectral type either.

One object, IRAS 01003-2238, may have an unusually low [N II] luminosity. The six line measurement methods for [N II] in this object are in reasonable agreement, despite the low $\mathrm{S} / \mathrm{N}$. We therefore do not have a good explanation for this. The only other way in which IRAS 01003-2238 is atypical is that its optical spectrum contains Wolf-Rayet star features (Farrah et al. 2005), but we do not see why this would affect the [N II] luminosity.

Finally, we comment briefly on two line ratios. First is $\left[\mathrm{O}_{\mathrm{I}}\right] 145 /\left[\mathrm{O}_{\mathrm{I}}\right] 63$, which is related to the physical conditions in PDRs. This ratio (note that the inverse is plotted in Figure 5) depends on both the gas temperature and density. It decreases with decreasing gas temperature, and decreases with increasing 


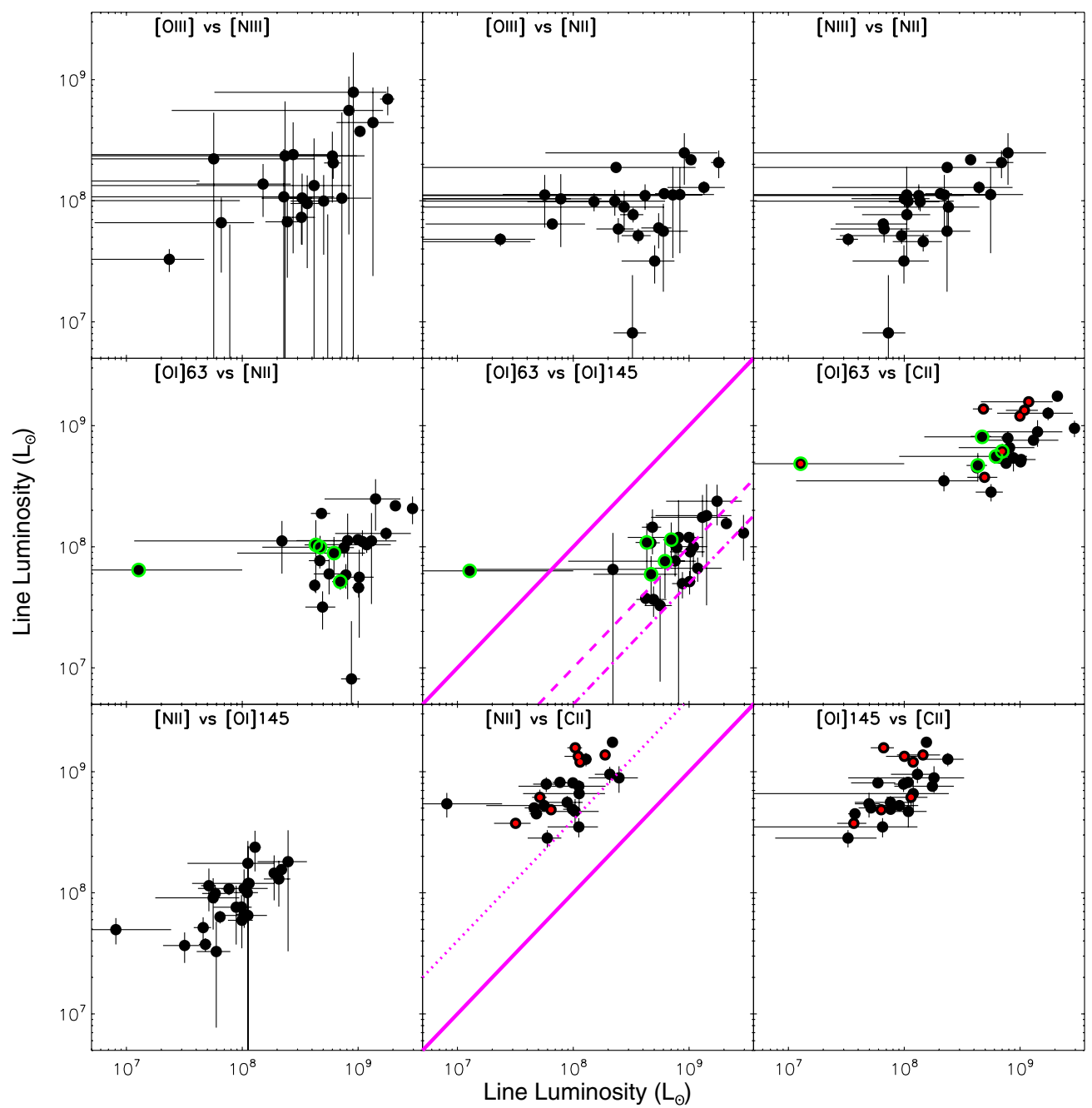

Figure 5. Selected far-IR line luminosities plotted against each other (Section 3.1.2). The label in each panel identifies which line is plotted on the $x$ and $y$ axes, respectively. The solid, dashed, and dot-dashed lines in the [O I]63 vs. [O I] 145 plot indicate ratios of 1:1, 10:1, and 20:1, respectively. The solid and dotted lines in the [N II] vs. [C II] plot indicate ratios of 1:1 and 1:4, respectively. Objects with green annuli may show self absorption in [O I]63. Objects with red cores may show an additional component in $[\mathrm{C} \mathrm{II}]$.

(A color version of this figure is available in the online journal.)

gas density if the density is above the critical density. In a diffuse PDR illuminated by intense UV radiation, the expected [O I] ratio lies below $\sim 0.1$ (e.g., Kaufman et al. 2006). From Figure 5, we see that several of our sample have [O I] ratios above 0.1 . A likely reason for this is the self absorption of [O I]63, or a higher optical depth in [O I]63 than [O I]145 (e.g., Abel et al. 2007).

Second is the $[\mathrm{N} \mathrm{II}] /[\mathrm{C} \mathrm{II}]$ ratio, which can be used to estimate the relative contribution of [C $\mathrm{II}]$ arising in PDRs and in the ionized gas. While $[\mathrm{NII}]$ arises almost entirely from $\mathrm{H}_{\mathrm{II}}$ regions, $\left[\mathrm{C}_{\mathrm{II}}\right]$ can additionally arise from PDRs and the diffuse ISM (cold neutral, warm neutral, and warm diffuse ionized). The $[\mathrm{N} \mathrm{II}] /[\mathrm{C} \mathrm{II}]$ ratio can therefore quantify the contribution to [C II] from the ionized gas by comparing the observed $[\mathrm{N}$ II] $/[\mathrm{C}$ II $]$ ratio with theoretical expectations from a photoionized nebula. The expected ratio in the ionized gas lies between 0.2 and 2.0 depending on density (e.g., Figure 7 of Bernard-Salas et al. 2012). The ratios in our sample, however, are nearly all below 0.25 . This indicates that a significant fraction of the [C II] emission originates from photodissociation regions (PDRs). A precise estimate, however, is hampered by our lack of knowledge on the ionized gas density.

\subsection{Infrared Luminosity}

\subsubsection{Line Luminosities}

We compare far-IR line luminosities to $L_{\mathrm{IR}}$ in Figure 7. Qualitatively, some of the line luminosities crudely correlate with $L_{\mathrm{IR}}$, with greater scatter among the Sy1s. To determine if correlations exist between $L_{\mathrm{IR}}$ and $L_{\mathrm{Line}}$, we assume that $L_{\mathrm{IR}}$ and $L_{\text {Line }}$ are related by

$$
\log _{10}\left(\frac{L_{\mathrm{IR}}}{L_{\odot}}\right)=\alpha+\beta \log _{10}\left(\frac{L_{\mathrm{Line}}}{L_{\odot}}\right)
$$

where $\alpha$ and $\beta$ are free parameters. We fit this relation following the method of Tremaine et al. (2002). For a correlation to exist, we require that $\beta>0$ at $>3.5 \sigma$ significance. We do not claim that Equation (2) codifies an underlying physical relation, or that Equation (2) is the only relation that applies over the luminosity range of our sample. ${ }^{18}$ Finally, we do not consider more complex models as our data do not prefer them.

\footnotetext{
18 Indeed, in most cases throughout this paper where fit results are quoted, a linear $(Y=\alpha+\beta X)$ or exponential $\left(Y=\alpha X^{\beta}\right)$ model with appropriate choices for $\alpha$ and $\beta$ both serve equally well.
} 


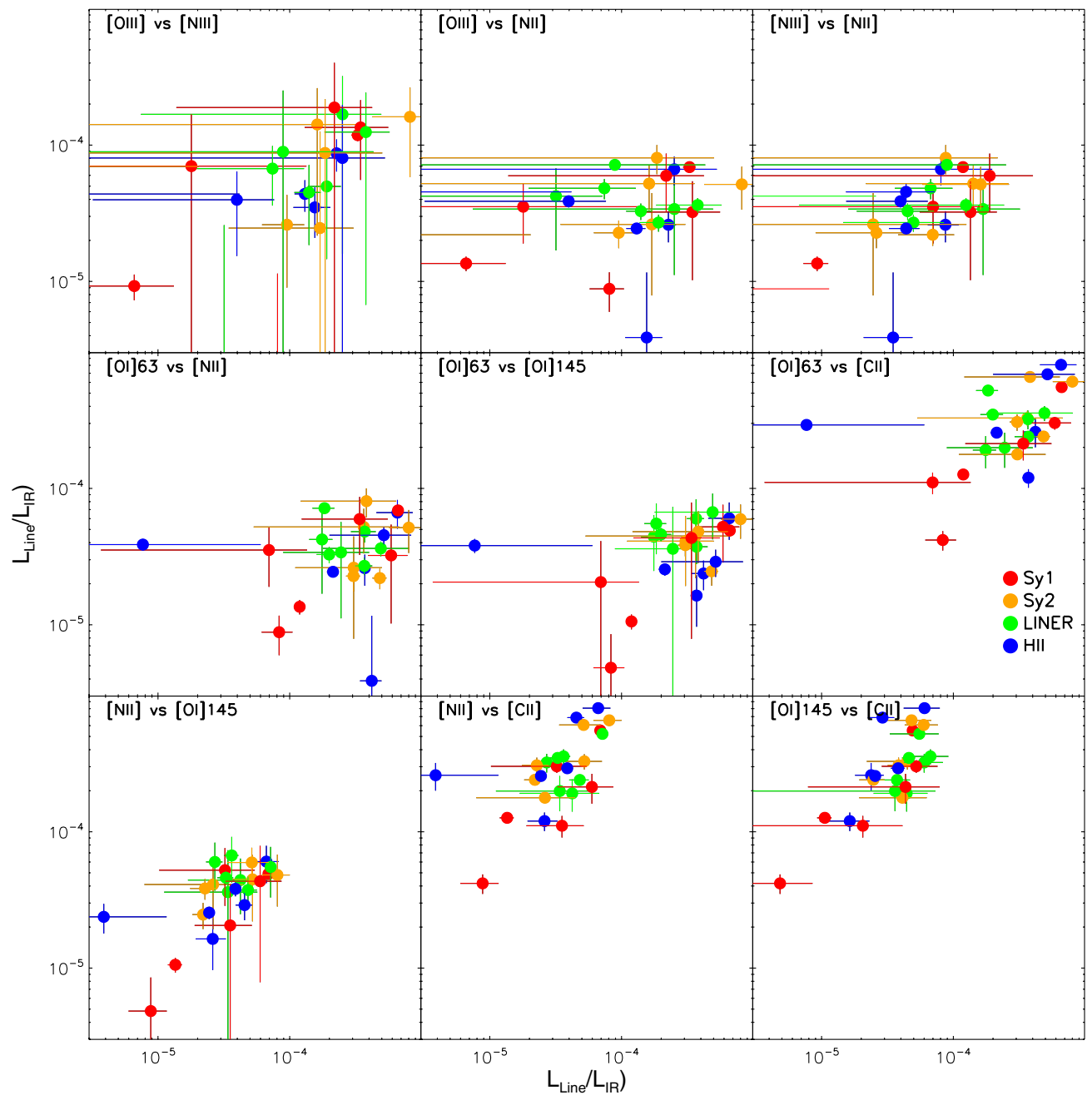

Figure 6. Selected far-IR line pairs normalized by $L_{\mathrm{IR}}$ plotted against each other (Section 3.1.2, see also Figure 5).

(A color version of this figure is available in the online journal.)

Excluding objects with Sy1 spectra yields the following relations between $L_{\mathrm{IR}}$ and $L_{\mathrm{Line}}$ :

$$
\begin{aligned}
\log _{10}\left(L_{\mathrm{IR}}\right) & =(4.46 \pm 1.77)+(0.92 \pm 0.20) \log _{10}\left(L_{[\mathrm{O} \mathrm{III}]}\right) \\
& =(4.74 \pm 1.59)+(0.94 \pm 0.19) \log _{10}\left(L_{[\mathrm{N} \mathrm{III}]}\right) \\
& =(4.28 \pm 1.89)+(0.91 \pm 0.21) \log _{10}\left(L_{[\mathrm{OI}] 63}\right) \\
& =(5.29 \pm 1.57)+(0.89 \pm 0.20) \log _{10}\left(L_{[\mathrm{N} \mathrm{III}}\right) \\
& =(1.75 \pm 2.11)+(1.34 \pm 0.27) \log _{10}\left(L_{[\mathrm{O} \mathrm{I}] 145}\right) \\
& =(6.73 \pm 2.44)+(0.64 \pm 0.27) \log _{10}\left(L_{[\mathrm{CII}]}\right),
\end{aligned}
$$

which are significant (in terms of $\beta$ ) for all of the lines except $[\mathrm{C}$ II]. However, there is the caveat that some of the relations are based on a small number of formal detections (Table 3). Including the Sy1s yields significant correlations only for [O III] and $[\mathrm{O} I] 145$, both with flatter slopes than the above. The insignificant correlation for [C II] suggests that it traces $L_{\mathrm{IR}}$ to an accuracy of about an order of magnitude at best. This result is consistent with the findings of previous authors, who find a crude correlation over a wider luminosity range (Sargsyan et al. 2012).

We also investigated whether sums of the lines in Equations (3a)-(3f) show stronger relations with $L_{\mathrm{IR}}$ than individual lines. We found correlations in several cases, but none that significantly improved on those for the individual lines.

The weaker correlations between $L_{\mathrm{IR}}$ and $L_{\text {Line }}$ when Sy1s are included in the fits are consistent with far-IR lines being better tracers of $L_{\mathrm{IR}}$ in ULIRGs without an optical AGN. If this is true, then we may see a similar result if we include in the fits only those objects with prominent PAH features, since PAHs are probably exclusively associated with star formation (see Section 3.3.1). We test this hypothesis by repeating the fits, but this time including only those objects with $11.2 \mu \mathrm{m}$ PAH EWs greater than $0.05 \mu \mathrm{m}$. We chose the $11.2 \mu \mathrm{m}$ PAH because (1) it is bright, and (2) its value correlates well with the evolutionary paradigm for ULIRGs in Farrah et al. (2009). ${ }^{19}$

\footnotetext{
${ }^{19}$ Using the $11.2 \mu \mathrm{m}$ PAH may, however, lead to more bias than using the $6.2 \mu \mathrm{m}$ PAH if absorption from ices and silicate dust is primarily associated with the background continuum source.
} 


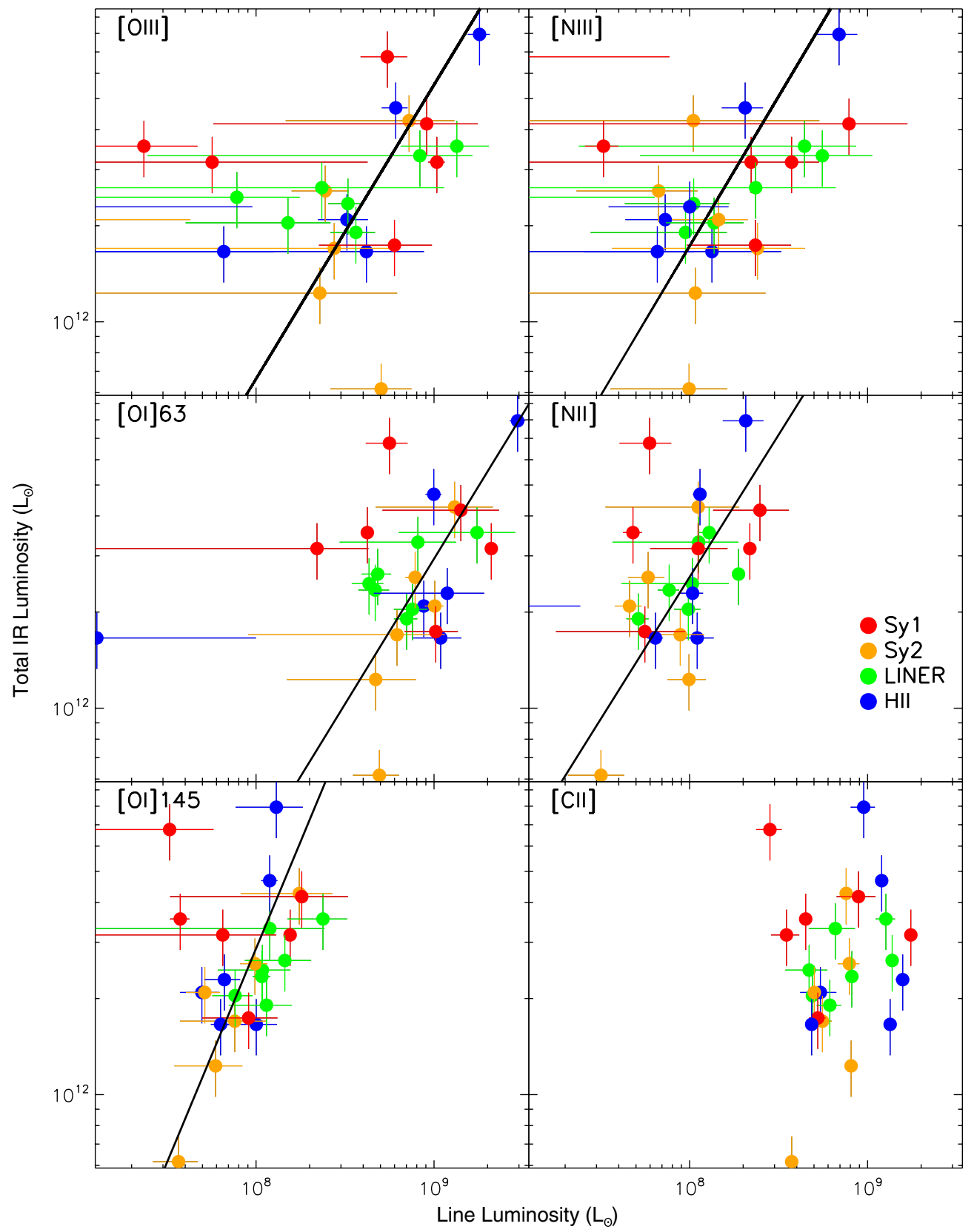

Figure 7. Far-IR line luminosities vs. $L_{\mathrm{IR}}$ (Section 3.2.1). The lines show the fits in Equations (3a)-(3f) if the fit is significant. Here and elsewhere, we use the same $x$ and $y$ axis ranges for each line, while still showing most of the objects and all significant trends.

(A color version of this figure is available in the online journal.)

We find

$$
\begin{aligned}
\log _{10}\left(L_{\mathrm{IR}}\right) & =(7.01 \pm 1.07)+(0.63 \pm 0.12) \log _{10}\left(L_{[\mathrm{O}} \mathrm{III}\right] \\
& =(6.52 \pm 1.33)+(0.71 \pm 0.16) \log _{10}\left(L_{[\mathrm{N} \mathrm{III}]}\right) \\
& =(6.71 \pm 1.30)+(0.64 \pm 0.15) \log _{10}\left(L_{[\mathrm{O} \mathrm{I}] 63}\right) \\
& =(6.43 \pm 1.51)+(0.74 \pm 0.19) \log _{10}\left(L_{[\mathrm{NII}}\right) \\
& =(2.77 \pm 2.12)+(1.20 \pm 0.26) \log _{10}\left(L_{[\mathrm{O} \mathrm{I}] 145}\right) \\
& =(9.44 \pm 2.20)+(0.33 \pm 0.25) \log _{10}\left(L_{[\mathrm{C} \mathrm{III}}\right) .
\end{aligned}
$$

These relations are somewhat flatter than those in Equations (3a)-(3f). Again, the [C $\mathrm{CI}]$ line shows no significant correlation. We conclude that these far-IR lines are better tracers of $L_{\mathrm{IR}}$ in systems without type $1 \mathrm{AGN}$, but that it is unclear whether they are better tracers of $L_{\mathrm{IR}}$ in systems with more prominent star formation.

\subsubsection{Continuum Luminosities}

We compare $L_{\mathrm{IR}}$ to continuum luminosity densities (in units of $L_{\odot} \mathrm{Hz}^{-1}$ ) near the wavelengths of the far-IR lines in Figure 8 . We find a significant correlation at all six wavelengths, irrespective of whether Sy1s are included (though the continua of the Sy1s are usually not detected). For consistency with the line comparisons, we exclude the Sy1s and fit relations of the 


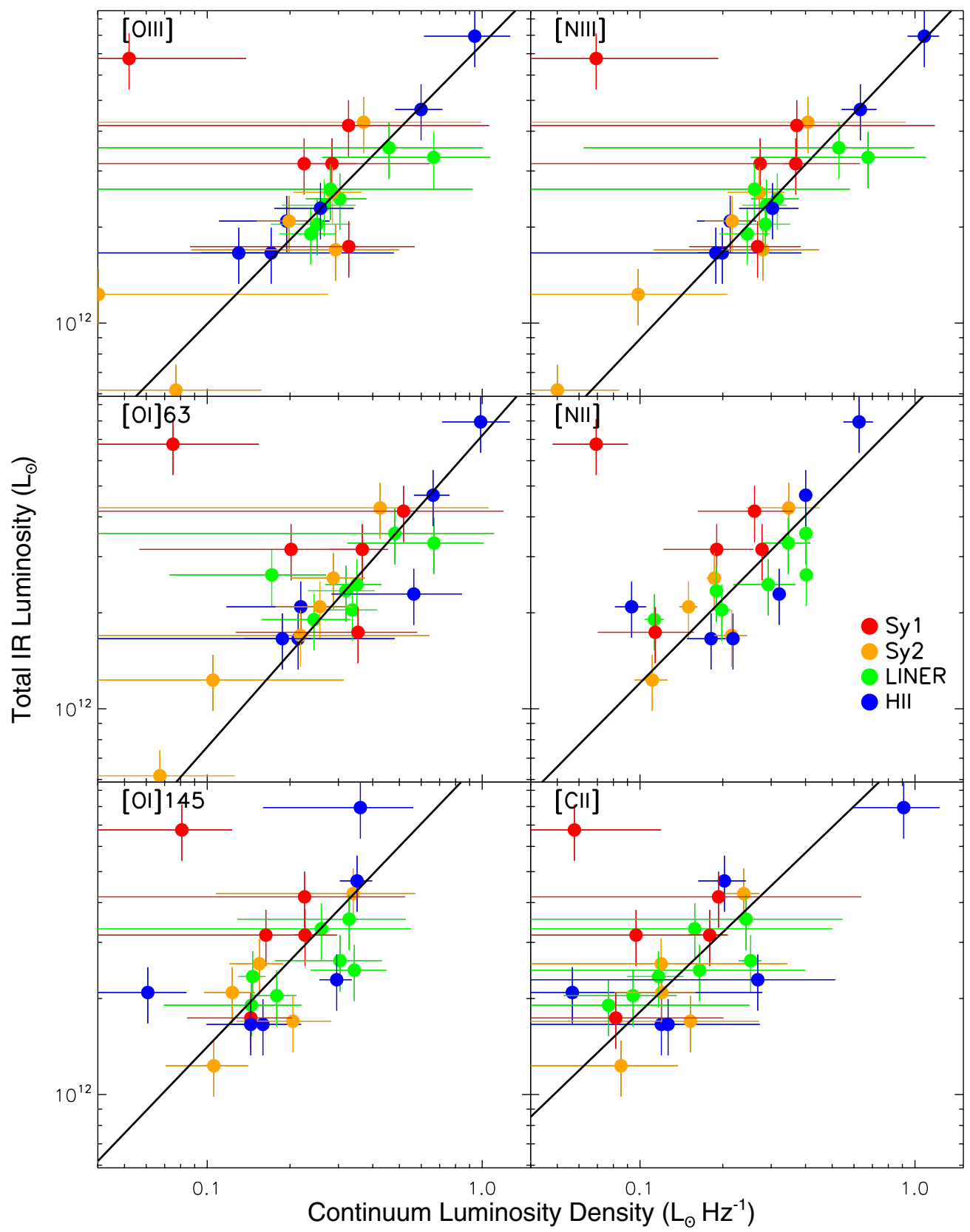

Figure 8. Continuum luminosity densities near the wavelength of the indicated line, vs. total IR luminosity (Section 3.2.2). The lines show fits with the Sy1s removed (Equations (5a)-(5f)). The Sy1 object with low flux densities near all six lines but a high IR luminosity is 3C 273.

(A color version of this figure is available in the online journal.)

form in Equation (2), obtaining

$$
\begin{aligned}
\log _{10}\left(L_{\mathrm{IR}}\right) & =(12.87 \pm 0.09)+(0.88 \pm 0.17) \log _{10}\left(L_{52 \mu \mathrm{m}}\right) \\
= & (12.86 \pm 0.05)+(0.91 \pm 0.11) \log _{10}\left(L_{57 \mu \mathrm{m}}\right) \\
= & (12.86 \pm 0.08)+(0.98 \pm 0.18) \log _{10}\left(L_{63 \mu \mathrm{m}}\right) \\
= & (12.95 \pm 0.07)+(0.87 \pm 0.11) \log _{10}\left(L_{122 \mu \mathrm{m}}\right) \\
= & (13.05 \pm 0.11)+(0.90 \pm 0.16) \log _{10}\left(L_{145 \mu \mathrm{m}}\right) \\
= & (13.08 \pm 0.11)+(0.83 \pm 0.11) \log _{10}\left(L_{158 \mu \mathrm{m}}\right) .
\end{aligned}
$$

Including the Sy1s yields comparable relations. If we instead exclude objects with PAH $11.2 \mu \mathrm{m} \mathrm{EW} \lesssim 0.05$, then we obtain consistent relations.

\subsubsection{Line Deficits}

Several far-IR lines in ULIRGs show a deficit in their $L_{\text {Line }} / L_{\mathrm{IR}}$ ratios compared to the ratios expected from systems with lower values of $L_{\mathrm{IR}}$ (e.g., Luhman et al. 2003). In contrast, high-redshift ULIRGs, at least for [O I]63 and [C II], do not show such pronounced deficits (Stacey et al. 2010; Coppin et al. 2012; D. Rigopoulou et al., in preparation). We examine if the [O I], [N II], and [C II] lines in our sample show such deficits in Figure 9 (we do not test the other lines as we lack archival data to compare to). We find a deficit in all four lines. Comparing 


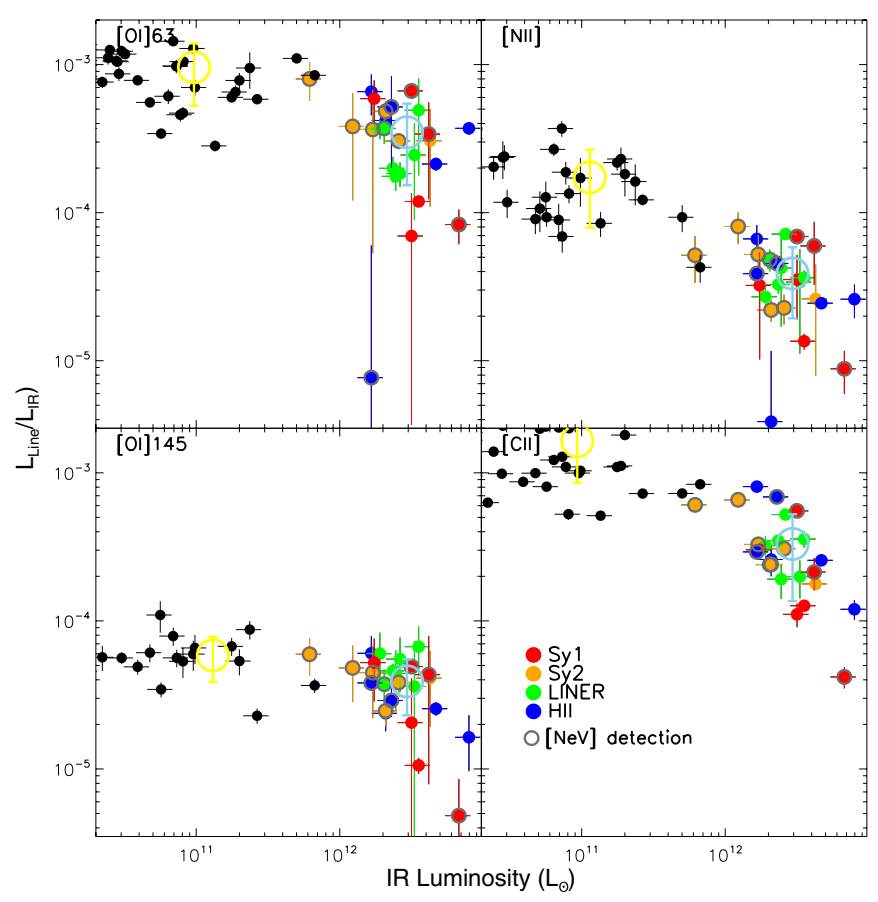

Figure 9. Comparison of the $\left[\mathrm{O}_{\mathrm{I}}\right] 63 / L_{\mathrm{IR}},\left[\mathrm{N}_{\mathrm{II}}\right] / L_{\mathrm{IR}},\left[\mathrm{O}_{\mathrm{I}}\right] 145 / L_{\mathrm{IR}}$, and [C II] $/ L_{\mathrm{IR}}$ ratios with $L_{\mathrm{IR}}$ (Section 3.2.3). The colored points are our sample, coded by optical class. ULIRGs with a gray annulus have a [Ne v]14.32 detection. The black points are taken from Brauher et al. (2008). The yellow and light blue open symbols show the means and dispersions for the Brauher and our samples, respectively. The ULIRGs show a deficit compared to the lower luminosity systems in all four cases. There is, however, no clear dependence of the deficit on optical class or the detection of [ $\mathrm{Ne} \mathrm{v}] 14.32$.

(A color version of this figure is available in the online journal.)

the mean ratios at $10^{11} L_{\odot}$ and $10^{12.2} L_{\odot}$, we find differences of factors of $2.75,4.46,1.50$, and 4.95 for [O I] 63 , [N II], [O I] 145 , and [C II], respectively.

The four line deficits show no clear dependence on optical spectral type, the presence of an obscured AGN (as diagnosed from the detection of [Ne v]14.32, see Section 3.5), or on PAH $11.2 \mu \mathrm{m}$ EW for any line. There are, however, trends with both $S_{\text {Sil }}$ and merger stage.

1. If $S_{\mathrm{Sil}} \gtrsim 1.4$, then the [C II] and [N II] deficits increase with increasing $S_{\mathrm{Sil}}$. However, there is no obvious trend of the [C II] and [N II] deficits with $S_{\mathrm{Sil}}$ at $S_{\mathrm{Sil}} \lesssim 1.4$ (top left panel of Figures 10 and 13). Conversely, the [O I] deficits show no trends with $S_{\text {Sil }}$.

2. We find no evidence that the $[\mathrm{N}$ II $]$ and $\left[\mathrm{O}_{\mathrm{I}}\right]$ deficits depend on merger stage, but the [C II] deficit is stronger, on average, in advanced mergers (classes IVb and V) than in earlystage mergers (classes IIIa through IVa, top right panel of Figure $10,{ }^{20}$ see also Diaz-Santos et al. 2013).

Finally, in the bottom row of Figure 10, we plot $L_{\left[\mathrm{C}_{\text {II }}\right]} / L_{\mathrm{IR}}$ against $L_{\mathrm{PAH}} / L_{\mathrm{IR}}$ as a function of merger stage and $S_{\mathrm{Sil}}$. We see consistent trends in both plots; ULIRGs in advanced mergers and with $S_{\mathrm{Sil}} \gtrsim 2$ have lower $L_{[\mathrm{CI}} / L_{\mathrm{IR}}$ and $L_{\mathrm{PAH}} / L_{\mathrm{IR}}$ ratios, compared to ULIRGs in early-stage stage mergers and with $S_{\text {Sil }} \simeq 1.4-2$.

We do not believe that the line deficits arise from missing an asymmetric or broad component (see Section 3.1.1), since

\footnotetext{
20 We also constructed the deficit plots as a function of merger stage using only those sources with $S_{\mathrm{Sil}}<1.4$. The [C II] deficit still strengthens with advancing merger stage, while no trends emerge with merger stage for the other lines.
}

such components are rare, or that the deficits arise due to selfabsorption, since we see no self absorption in [C II], which shows the strongest deficit. We do, however, see self absorption in [O I]63, which shows a weaker deficit. Instead, the stronger $[\mathrm{C}$ II $]$ and $\left[\mathrm{N}\right.$ II] deficits in sources with higher $S_{\mathrm{Sil}}\left(\right.$ at $\left.S_{\mathrm{Sil}} \gtrsim 1.4\right)$ are consistent with the HII regions in ULIRGs being dustier than $\mathrm{H}$ II regions in lower luminosity systems. In this scenario (Luhman et al. 2003; González-Alfonso et al. 2008; Abel et al. 2009; Graciá-Carpio et al. 2011), a higher fraction of the UV photons are absorbed by dust rather than neutral Hydrogen, thus contributing more to $L_{\mathrm{IR}}$ but less to the photoionization heating of gas in the Hil regions, and thus decreasing line emission relative to $L_{\mathrm{IR}}$. This mechanism would also produce a deficit in [C II] and the "deficit" in the PAH emission, even if the bulk of the [C II] and PAHs are in the PDRs, since there would also be fewer UV photons for photoelectric heating of the PDRs. Furthermore, this mechanism is consistent with the [O I] deficits. From Figure 3 of Graciá-Carpio et al. 2011, we see that the conditions consistent with the observed deficits of all four lines are $n_{h} \lesssim 300 \mathrm{~cm}^{-3}$ and $0.01 \lesssim\langle U\rangle \lesssim 0.1$.

However, we propose that dustier $\mathrm{H}$ II regions are not the sole origin of the line deficits. This is based on three observations. First, it is puzzling that we see no strong dependence of any of the line deficits on $S_{\text {Sil }}$ when $S_{\text {Sil }} \lesssim 1.4$; if the deficit arises entirely in $\mathrm{H}$ II regions, and if $S_{\mathrm{Sil}}$ is a proxy for the dust column in these $\mathrm{H}$ II regions at $S_{\mathrm{Sil}} \gtrsim 0$, then we should see a dependence. We note, however, that we have only a small sample, so we could be missing a weak dependence, and the assumption that $S_{\text {Sil }}$ is a proxy for the dust column in H II regions is not proven. ${ }^{21}$ Second, we see significant line deficits for some sources with $S_{\text {Sil }}<0$, i.e., a silicate emission feature. Third, only the [C II] deficit becomes stronger with advancing merger stage. If more advanced mergers host dustier $\mathrm{H}$ II regions, then we would also see a dependence of the $[\mathrm{N}$ II] deficit on merger stage.

These observations suggest that some fraction of the [C II] deficit is not driven by increased dust in $\mathrm{H}$ II regions. We lack the data to investigate this in detail, so we only briefly discuss this further. We consider three possible origins: (1) increased charging of dust grains (e.g., Malhotra et al. 2001), leading to a lower gas heating efficiency, (2) a softer radiation field in the diffuse ISM (e.g., Spaans et al. 1994), and (3) dense gas in the PDRs, making [O I] the primary coolant rather than [C II].

The third of these possibilities is feasible, but we do not have the data to confirm or refute it as a mechanism. The second possibility is unlikely, due to the energetic nature of star formation in ULIRGs and considering the arguments in Luhman et al. (2003). For the first possibility, if the origin of the additional deficit in [C II] is grain charging, then we would see a higher $G_{0}$ in advanced mergers compared to early stage mergers. The advanced mergers have roughly an order of magnitude higher value of $G_{0}$ for about the same $n$ (see Section 3.6). ${ }^{22}$ We therefore infer, cautiously and with the caveat that we cannot rule out [O I] being a major cooling line, that part of the [C II] deficit arises due to grain charging in PDRs or in the diffuse ISM. We further propose that this increase in grain charge is not driven mainly by a luminous AGN, obscured or otherwise, since we see no

\footnotetext{
21 It is also (arguably) puzzling that we see dependences on any line deficit at $S_{\text {Sil }} \gtrsim 1.4$, since (some) models demand that silicate strengths greater than about this value require smooth rather than clumpy dust distributions (Nenkova et al. 2008).

22 We note though that this also holds for the trends discussed with $S_{\mathrm{Sil}}$; dividing into two samples with $1.4<S_{\text {Sil }}<2.1$ and $S_{\text {Sil }}>2.1$ yields a $G_{0} / n$ ratio about a factor of three higher in the latter sample.
} 

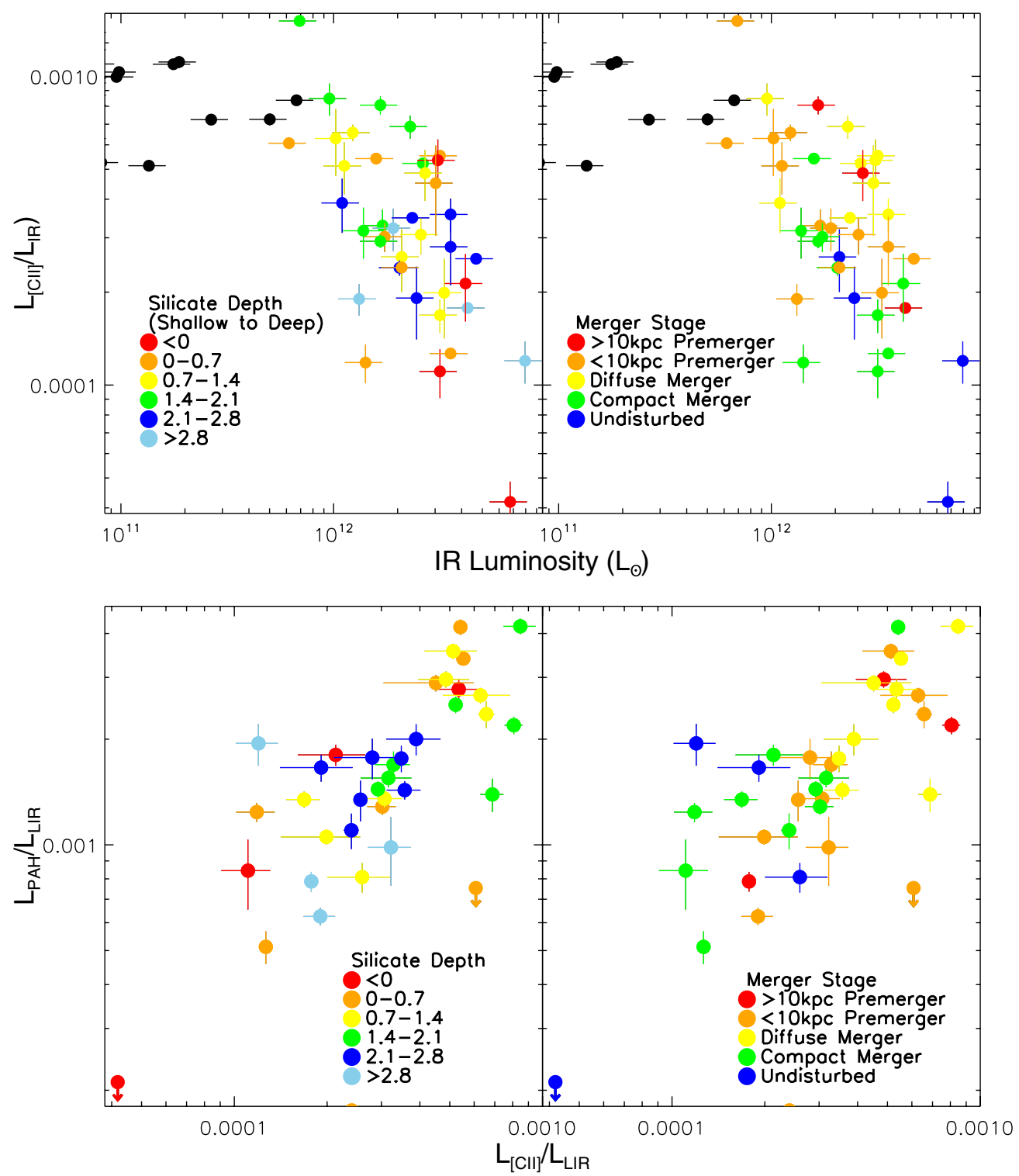

Figure 10. Top row: zoom in of the $[\mathrm{C}$ II $] / L_{\mathrm{IR}}$ deficit in ULIRGs as a function of merger stage and silicate depth (Section 3.2.3). Other details are as in Figure 9. We include in this plot additional ULIRGs with [C II] detections: Arp220, Mrk 273, NGC 6240, IRAS 04103-2838, IRAS 05189-2524, IRAS 10565+2448, IRAS 12018+1941, IRAS 13342+3932, IRAS 15001+1433, IRAS 17208-0014, IRAS 20037-1547, IRAS 20100-4156, IRAS 20551-4250, and IRAS 23128-5919 (Brauher et al. 2008). Bottom row: [C II $] / L_{\mathrm{IR}}$ plotted against $L_{\mathrm{PAH}} / L_{\mathrm{IR}}$.

(A color version of this figure is available in the online journal.)

dependence of the $[\mathrm{C}$ II] deficit on either optical spectral type or the presence of $[\mathrm{Ne} \mathrm{v}] 14.32$.

\subsection{Star Formation Rate}

\subsubsection{Line Luminosities}

We examine far-IR fine-structure lines as star formation rate indicators by comparing their luminosities to those of PAHs. PAHs are thought to originate from short-lived Asymptotic Giant Branch stars (Gehrz 1989; Habing 1996; Blommaert et al. 2005; Bernard-Salas et al. 2006), and therefore to be associated with star-forming regions (Tielens 2008). They are prominent in starburst galaxies but weak in AGNs (Laurent et al. 2000; Weedman et al. 2005). While we are still uncertain how to calibrate PAHs as star formation rate measures (Peeters et al. 2004; Förster Schreiber et al. 2004; Sargsyan et al. 2012), their luminosities are likely reasonable proxies for the instantaneous rate of star formation. Conversely, for far-IR fine-structure lines, the relation between line luminosity and star formation rate is less clear. We may expect a correlation, as the inter-stellar radiation field (ISRF) from young stars may be an important excitation mechanism. Moreover, correlations between far-IR line luminosities and the star formation rate have been observed previously (e.g., Boselli et al. 2002; de Looze et al. 2011; Sargsyan et al. 2012; Zhao et al. 2013). The extent to which correlations exist is, however, poorly constrained.

Considering the caveats that PAH luminosity depends on both metallicity (Madden et al. 2006; Calzetti et al. 2007; Khramtsova et al. 2013) and dust obscuration, neither of which we can correct for, we assume that the PAH luminosities give "true" star formation rate measures, which we compare to the farIR line luminosities. In doing so, we further assume that there is negligible differential extinction between the PAHs and the 


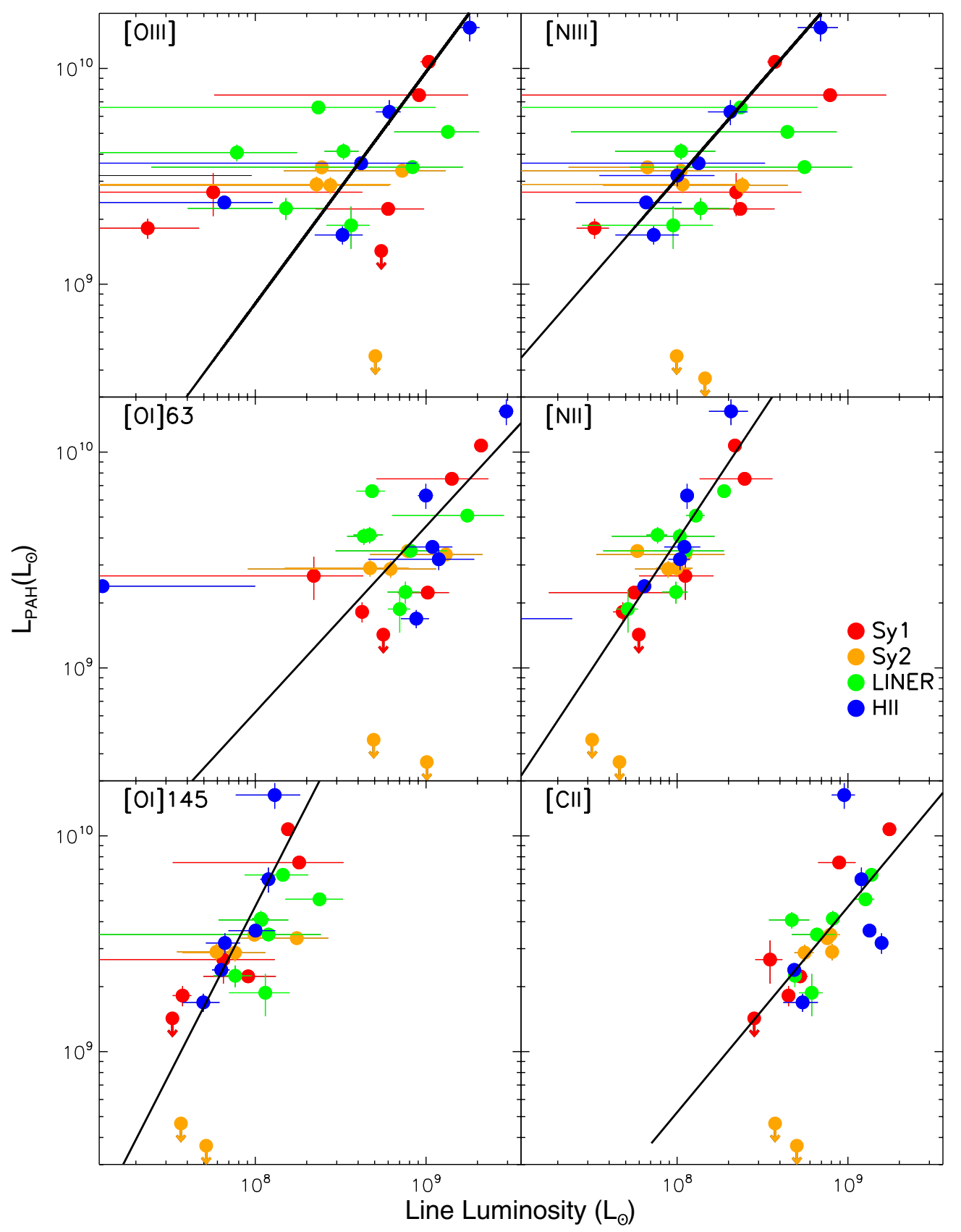

Figure 11. Far-IR line luminosities vs. $L_{\mathrm{PAH}}$ (Section 3.3.1). The black lines show the fits in Equations (6a)-(6f).

(A color version of this figure is available in the online journal.)

far-IR lines. To mitigate effects from the variation of individual PAH features (Smith et al. 2007), we sum the luminosities of the PAH $6.2 \mu \mathrm{m}$ and $11.2 \mu \mathrm{m}$ features into a single luminosity, $L_{\mathrm{PAH}}$ (Farrah et al. 2007a).

We plot far-IR line luminosities against $L_{\mathrm{PAH}}$ in Figure 11. Unlike the plots with $L_{\mathrm{IR}}$, there is no significant difference between the Sy1s and HII/LINERs. This is consistent with both PAHs and the far-IR lines primarily tracing star formation. Fitting relations of the form in Equation (2) to all the objects and converting to star formation rate by using Equation (5) of Farrah et al. (2007a) yields

$$
\begin{aligned}
\log _{10}\left(\frac{\dot{M}_{\odot}}{M_{\odot} \mathrm{yr}^{-1}}\right)= & (-7.02 \pm 1.25) \\
& +(1.07 \pm 0.14) \log _{10}\left(L_{[\mathrm{O}} \mathrm{III}\right]
\end{aligned}
$$

$$
\begin{aligned}
& =(-5.13 \pm 0.72)+(0.91 \pm 0.09) \log _{10}\left(L_{[\mathrm{N} \mathrm{III]}}\right) \\
& =(-5.44 \pm 1.79)+(0.86 \pm 0.20) \log _{10}\left(L_{[\mathrm{O} \mathrm{I}] 63}\right) \\
& =(-7.30 \pm 0.87)+(1.19 \pm 0.11) \log _{10}\left(L_{[\mathrm{N} \mathrm{III}]}\right) \\
& =(-10.04 \pm 1.34)+(1.55 \pm 0.17) \log _{10}\left(L_{[\mathrm{O} \mathrm{I}] 145}\right) \\
& =(-6.24 \pm 1.72)+(0.95 \pm 0.19) \log _{10}\left(L_{\left[\mathrm{C}_{\mathrm{II}}\right]}\right) .
\end{aligned}
$$

Using the criteria from Section 3.2.1, all of the lines show a significant correlation. The trends with $\left[\mathrm{O}_{\mathrm{I}}\right] 63$ and $\left[\mathrm{C}_{\mathrm{II}}\right]$ 


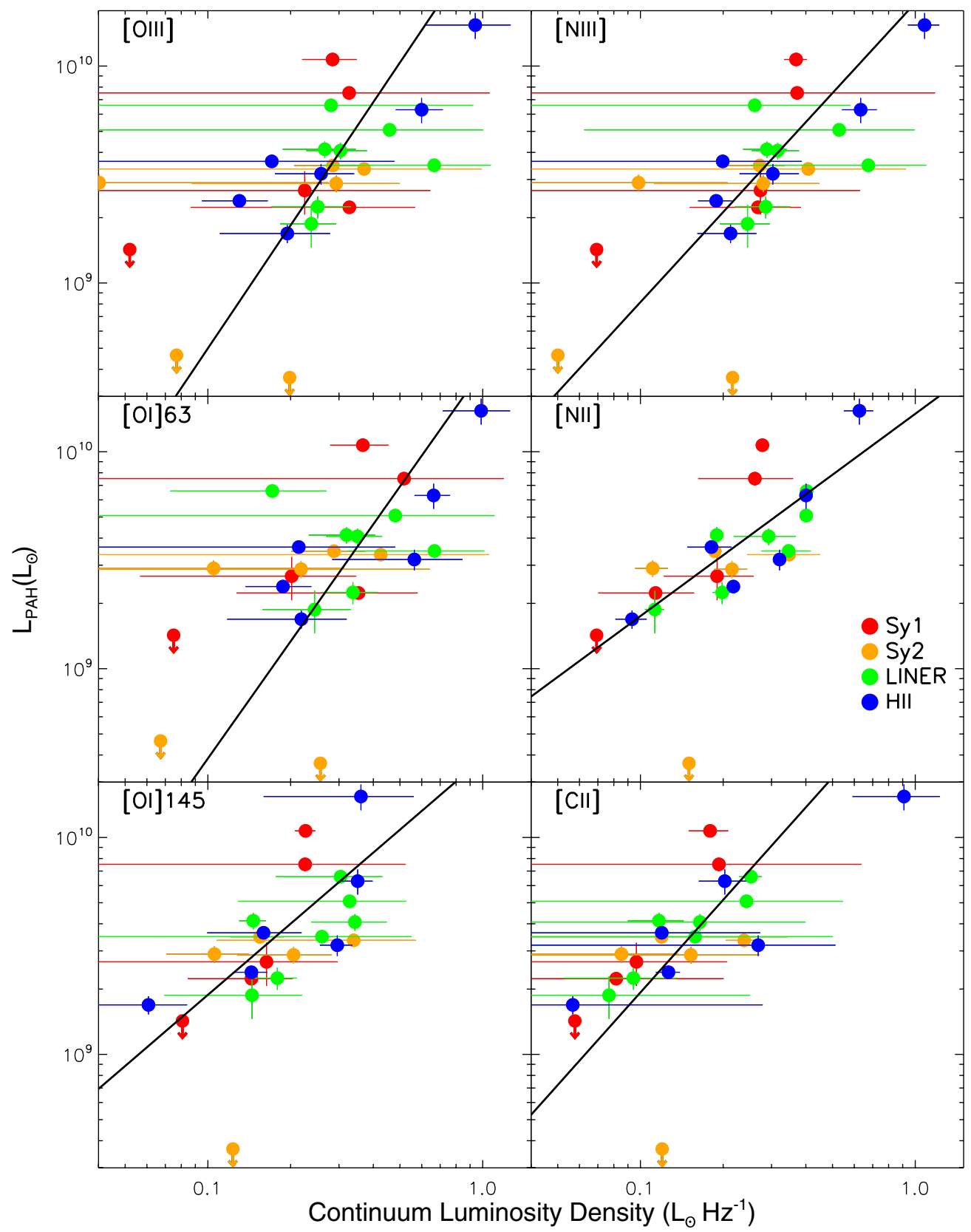

Figure 12. Continuum luminosity densities at the wavelength of the indicated line vs. $L_{\mathrm{PAH}}$ (Section 3.3.2). The fits are given in Equations (8a)-(8f).

(A color version of this figure is available in the online journal.)

are only barely significant (see also Diaz-Santos et al. 2013). Excluding the Sy1s and Sy2s from the fits yields consistent slopes and intercepts in all cases, though the fits are now no longer significant for [O I $] 63$ and $\left[\mathrm{C}_{\mathrm{II}}\right]$. Considering only those lines detected at $>3 \sigma$, the derived star formation rates for a given object are consistent to within a factor of three in nearly all cases. We present the mean star formation rates in Table 4.

An alternative to PAH luminosity as a tracer of star formation rate is the sum of the [Ne III] $15.56 \mu \mathrm{m}$ and [Ne II] $12.81 \mu \mathrm{m}$ line luminosities, $L_{\text {Neon }}$ (Thornley et al. 2000; Ho \& Keto 2007; Shipley et al. 2013). We thus compared $L_{\text {Neon }}$ to $L_{\text {Line. }}$ For the whole sample, we find relations that are mostly in agreement with Equations (6a)-(6f), though the relation with [C II] is now formally insignificant. Excluding the Seyferts and comparing $L_{\text {Neon }}$ to $L_{\text {Line }}$ yields similar results to those with $L_{\mathrm{PAH}}$.
We note four additional points. First, we tested sums of far-IR lines as tracers of star formation rate but found no improvement on the individual relations. Second, if we instead consider $L_{\mathrm{Line}} / L_{\mathrm{IR}}$ versus $L_{\mathrm{PAH}} / L_{\mathrm{IR}}$, then we see correlations with comparable scatter to those in Figure 11. Third, we investigated whether using $L_{\mathrm{PAH}}$ is better than using a single PAH luminosity by reproducing Figure 11 using only the PAH $6.2 \mu \mathrm{m}$ luminosity. We found consistent relations in all cases, albeit with larger scatter for the $L_{\mathrm{PAH}}^{6.2}$ plots. Fourth, if we instead code the points in Figure 11 by the $11.2 \mu \mathrm{m}$ PAH EW, then we see no dependence of the relations on the energetic importance of star formation.

Finally, we note two points about the relation between star formation rate and $L_{[\mathrm{CII}]}$. First, we investigated whether $L_{[\mathrm{CI}]}$ shows an improved correlation with star formation rate if objects with a strong [C II] deficit are excluded. Adopting a (somewhat 


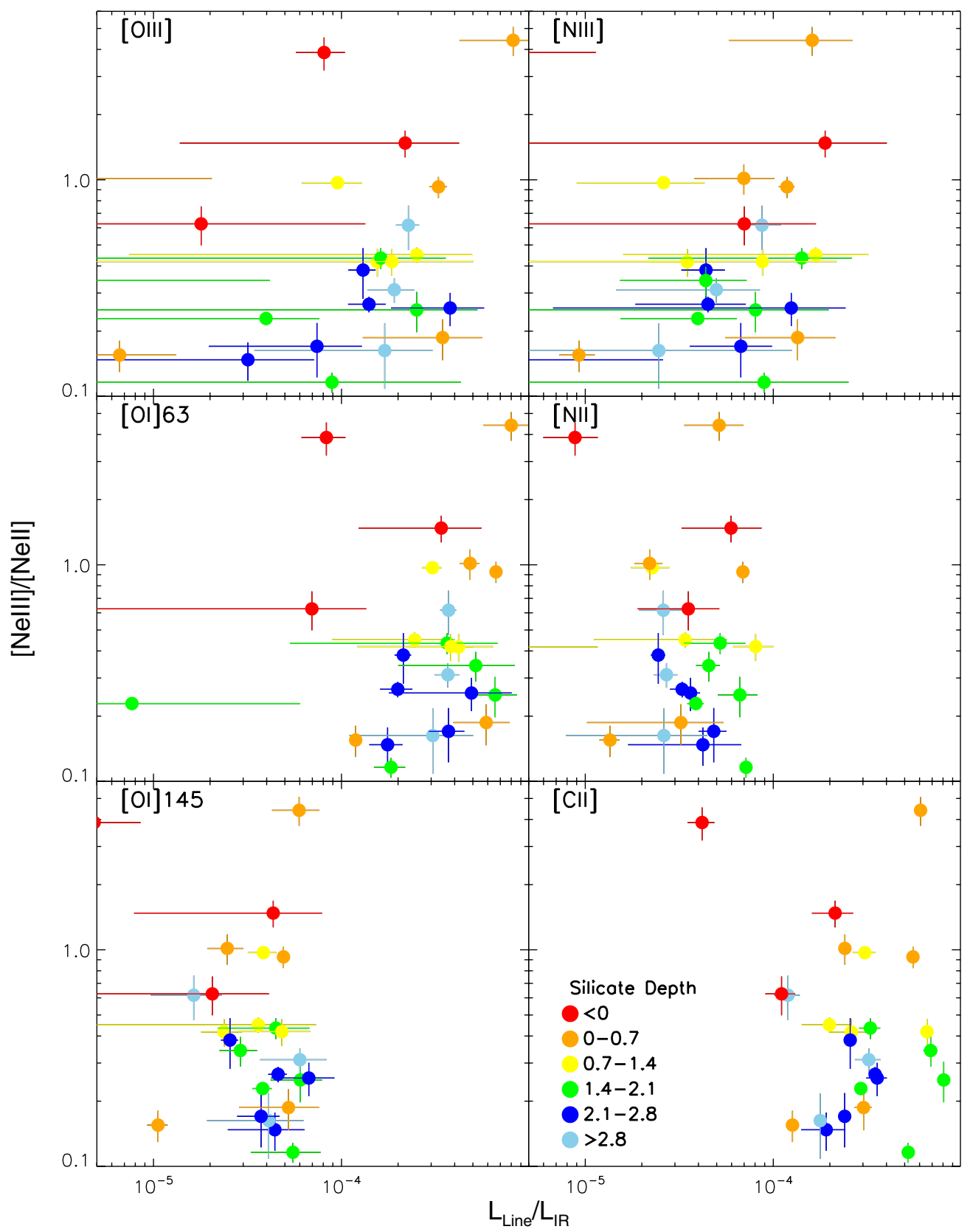

Figure 13. Normalized far-IR line luminosities plotted against [Ne III] 15.56/[Ne II]12.81. There are no clear correlations (Section 3.4). We code the points by $S_{\text {Sil }}$ to highlight the trends described in Section 3.2.3.

(A color version of this figure is available in the online journal.)

arbitrary) boundary of $L_{[\mathrm{CII}]} / L_{\mathrm{IR}}=2 \times 10^{-4}$ yields only a marginally different relation:

$$
\begin{aligned}
\log _{10}\left(\frac{\dot{M}_{\odot}}{M_{\odot} \mathrm{yr}^{-1}}\right)= & (-6.59 \pm 1.58) \\
& +(0.99 \pm 0.18) \log _{10}\left(L_{[\mathrm{C} \text { II] }}\right),
\end{aligned}
$$

suggesting that the correlation does not depend strongly on the [C II] deficit, though there is the caveat that star formation rate is derived from $L_{\mathrm{PAH}}$ (see Section 3.2.3 and Figure 10). Second, the relation between $\dot{M}_{\odot}$ and $L_{[\mathrm{C} \text { II] }}$ is consistent with that given by Sargsyan et al. (2012), though their sample mostly consists of lower luminosity systems.

\subsubsection{Continuum Luminosities}

We examine continuum luminosity densities as star formation rate tracers in Figure 12. Employing the same method as in Section 3.2, we find that the continua near all the lines provide acceptable fits. Converting to relations with star formation rate (see Section 3.3), we find

$$
\begin{array}{r}
\log _{10}\left(\frac{\dot{M}_{\odot}}{M_{\odot} \mathrm{yr}^{-1}}\right)=(3.24 \pm 0.24)+(1.89 \pm 0.46) \log _{10}\left(L_{52 \mu \mathrm{m}}\right) \\
\quad=(2.95 \pm 0.10)+(1.38 \pm 0.19) \log _{10}\left(L_{57 \mu \mathrm{m}}\right) \\
\quad=(3.04 \pm 0.17)+(1.79 \pm 0.39) \log _{10}\left(L_{63 \mu \mathrm{m}}\right)
\end{array}
$$




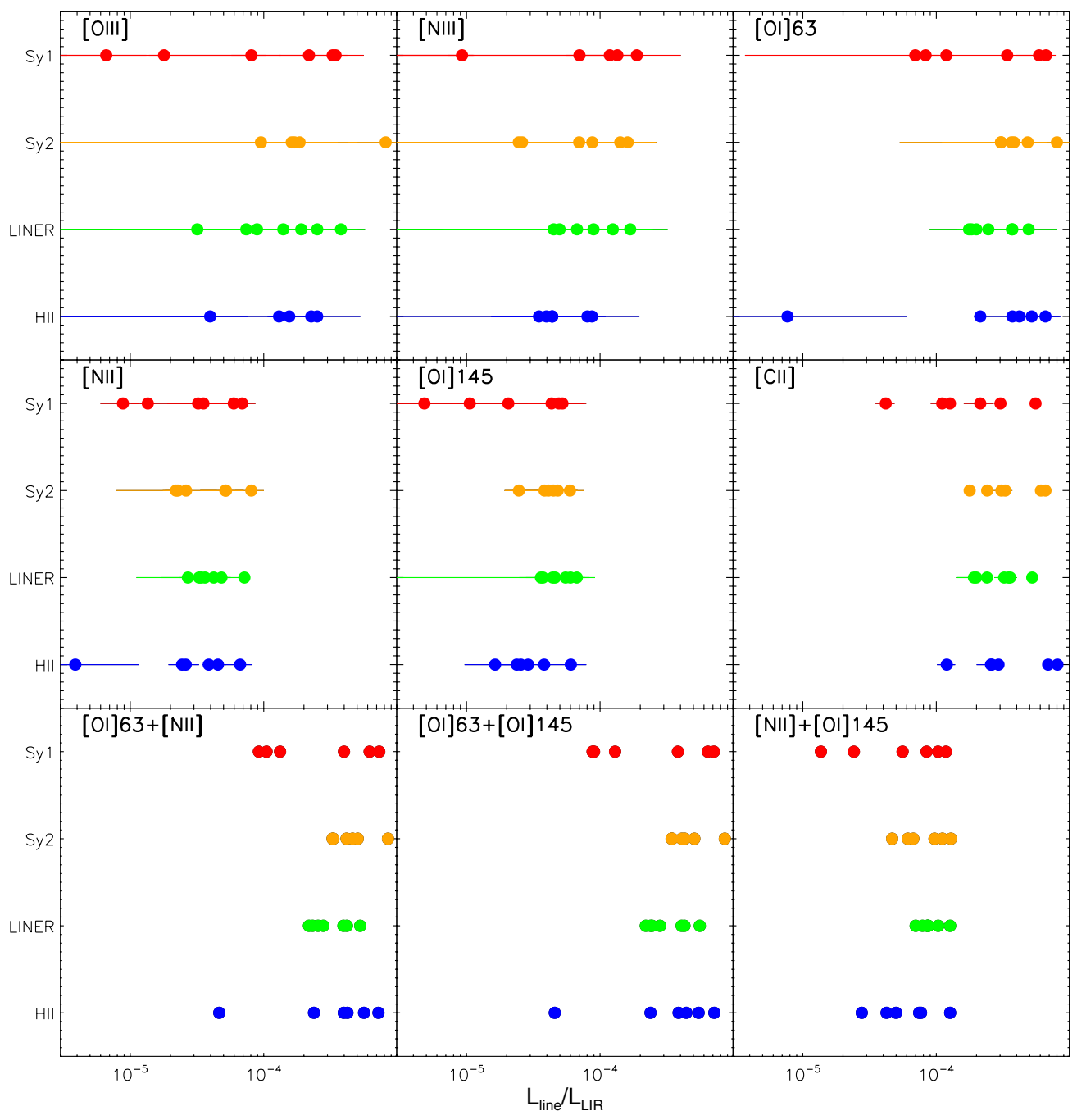

Figure 14. Normalized far-IR line luminosities vs. optical class (Section 3.5).

(A color version of this figure is available in the online journal.)

$$
\begin{aligned}
& =(2.83 \pm 0.11)+(0.93 \pm 0.16) \log _{10}\left(L_{122 \mu \mathrm{m}}\right) \\
& =(3.02 \pm 0.18)+(1.09 \pm 0.25) \log _{10}\left(L_{145 \mu \mathrm{m}}\right) \\
& =(3.36 \pm 0.22)+(1.42 \pm 0.30) \log _{10}\left(L_{158 \mu \mathrm{m}}\right),
\end{aligned}
$$

which are all significant using the criteria from Section 3.2.1. However, the correlations with continua at $\leqslant 63 \mu \mathrm{m}$ may be stronger, which is consistent with stronger correlations with warmer (star formation heated) dust. This is consistent with findings by previous authors (Brandl et al. 2006; Calzetti et al. 2007). We present the mean star formation rates derived from these relations in Table 4. In most cases, they are consistent with the line-derived star formation rates.

\subsection{Gas Photoionization}

If electron densities are below the critical density in the narrow-line region, then the hardness of the radiation field ionizing an element can be estimated via the flux ratios of adjacent ionization states of that element: $f_{X^{i+1}} / f_{X^{i}}$. For a fixed $U$ this ratio will be approximately proportional to the number of photons producing the observed $X^{i}$ flux relative to the number of Lyman continuum photons.

For the mid-IR line-emitting gas, two diagnostic ratios of this type can be used: [Ne III] 15.56/[Ne II]12.81 and [S IV]10.51/[S III]18.71. The photon energies required to produce these four ions are all $<50 \mathrm{eV}$, meaning that they can be produced in star-forming regions (Smith \& Houck 2001; Bernard-Salas et al. 2001; Peeters et al. 2002; Verma et al. 2003). As the Neon lines are detected in all of our sample, we use the Neon ratio as a proxy for mid-IR gas excitation. ${ }^{23} \mathrm{We}$ find no trend of this ratio with individual far-IR line luminosities (e.g., Figure 13), either for the whole sample or for subsamples divided by optical type or PAH $11.2 \mu \mathrm{m}$ EW.

We also examined three mid-to-far and far-IR line ratios to try fashioning an excitation plane diagram, in a similar manner to Dale et al. (2006): [O IV]26/[O III], [N III]/[N II], and $[\mathrm{O}$ III $] /[\mathrm{N}$ II $]$. In no case did we find any trends. However, the large uncertainties on the [O IV $] 26$, [O III], and [N III] lines means that we cannot conclude that such trends do not exist.

\footnotetext{
${ }^{23}$ However, this ratio is unlikely to be a pure star formation tracer due to potential contamination of the [Ne III] $15.56 \mu \mathrm{m}$ flux by AGN (Gorjian et al. 2007).
} 


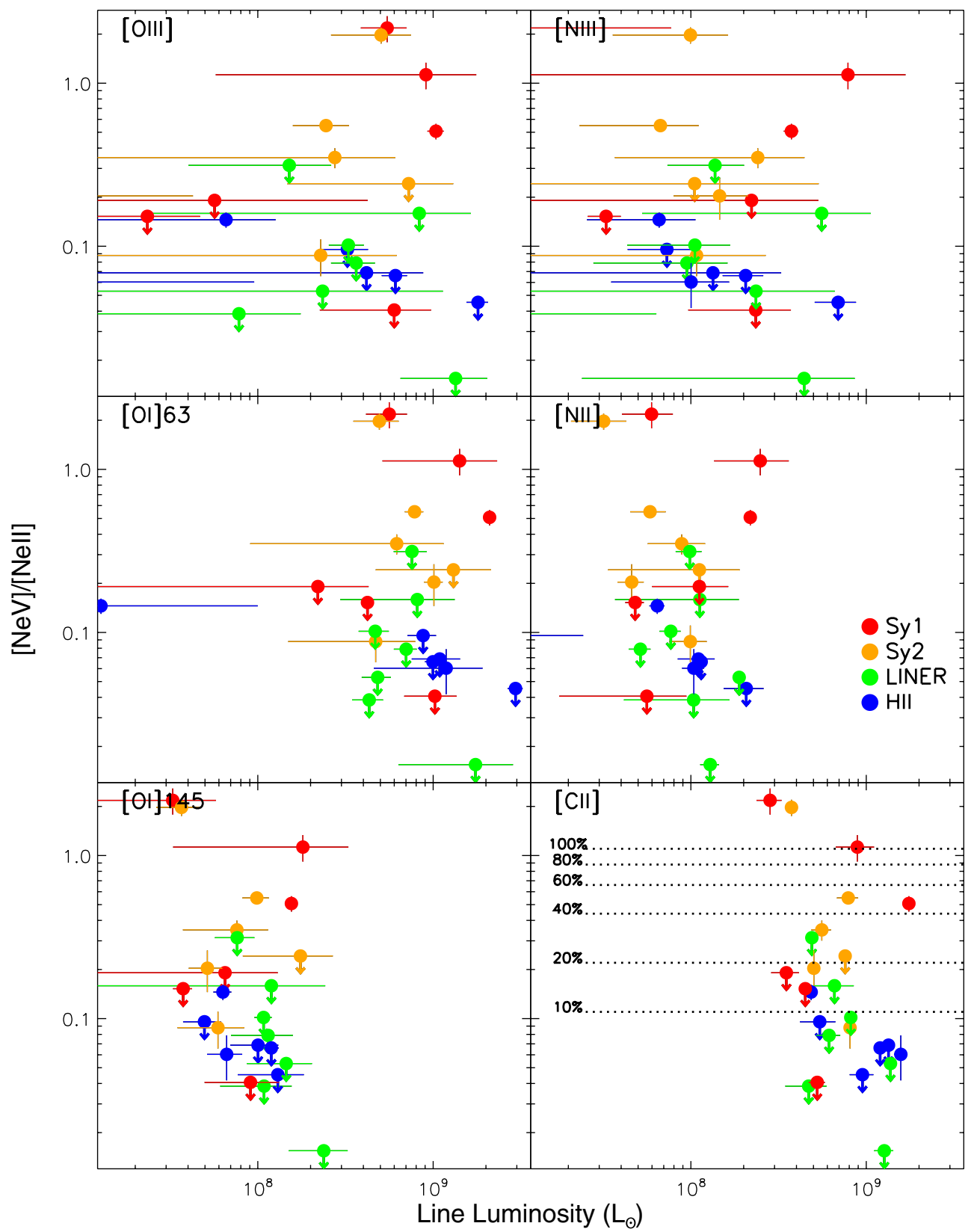

Figure 15. Far-IR line luminosities vs. [Ne v]14.32/[Ne II]12.88 (Section 3.5). The lines in the lower right panel are AGN contribution as a function of [Ne v]14.32/[Ne II] 12.88, from Sturm et al. 2002 (we plot these on only one panel for clarity).

(A color version of this figure is available in the online journal.)

\subsection{AGN Activity}

We first compare far-IR line luminosities to optical spectral classification and see no trends. If we normalize the line luminosities by $L_{\mathrm{IR}}$, then no trends emerge, either for individual lines or sums of lines (Figure 14). We also don't see any trends if we compare optical class to line ratios or normalized ratios. Moreover, the five objects with an additional broad component in [C II] (Section 3.1.1) do not have an unusually high incidence of Seyfert spectra. We conclude that optical class cannot be inferred from far-IR line luminosities or ratios. This is consistent with the gas producing the optical emission not being strongly associated (in terms of heating mechanism) with the far-IR line emitting gas, at least in the majority of cases.

Optical spectra may, however, misclassify AGN in obscured systems as H II or LINERs. We therefore employ the
$[\mathrm{Ne} \mathrm{v}] 14.32 /[\mathrm{Ne}$ II $] 12.88$ line ratio as an AGN diagnostic. Both of these lines are less affected by extinction than are optical lines. The $[\mathrm{Ne} \mathrm{v}] 14.32$ line can arise in planetary nebulae and supernova remnants (Oliva et al. 1999). For extragalactic sources though, this line is weak or absent in star forming regions (e.g., Lutz et al. 1998; Sturm et al. 2002; Bernard-Salas et al. 2009) but strong in the spectra of AGNs (e.g., Spinoglio et al. 2009). The [Ne II]12.88 line on the other hand is seen almost universally in galaxies. Their ratio should therefore be a reasonable proxy for the presence of an AGN.

We plot $[\mathrm{Ne}$ V] $14.32 /[\mathrm{Ne}$ II] 12.88 against far-IR line luminosities in Figure 15. There is a correlation between the Neon line ratio and optical classification, but no correlations with far-IR line luminosity. If we substitute optical class for PAH $11.2 \mu \mathrm{m} \mathrm{EW}$, then no trends emerge. Considering the Sturm et al. mixing lines (bottom right panel of Figure 15), we see no 
trends among objects classified either as weak or strong AGN. Finally, if we instead plot line luminosity normalized by $L_{\mathrm{IR}}$ on the $x$ axis, then we still do not see any trends.

We searched for trends with far-IR line ratios, normalized ratios, sums, and normalized (by $L_{\mathrm{IR}}$ ) sums, but found nothing convincing, though the small number of sources with $[\mathrm{Ne} \mathrm{v}] 14.32$ detections means we are not certain that no trends exist. We conclude, cautiously, that for ULIRGs there is no reliable diagnostic of AGN luminosity using only simple combinations of far-IR line luminosities. This result is consistent with the weaker correlation observed between $L_{\text {Line }}$ and $L_{\mathrm{IR}}$ if Sy1s are included (see Section 3.2.1), if the AGN supplies an effectively random additional contribution to $L_{\mathrm{IR}}$, thus increasing the scatter in the relation.

\subsection{UV Intensity and Electron Density}

From Sections 3.3 and 3.5 , it is plausible that at least the plurality of the $[\mathrm{CII}]$ emission arises from PDRs. We defer rigorous modeling to a future paper, and here only estimate the beam-averaged PDR hydrogen nucleus density, $n\left(\mathrm{~cm}^{-3}\right)$, and incident far-ultraviolet (FUV; $6 \mathrm{eV}<E<13.6 \mathrm{eV}$ ) radiation field intensity, $G_{0}$ (in units of the local Galactic interstellar FUV field found by Habing 1968; $\left.1.6 \times 10^{-3} \mathrm{erg} \mathrm{cm}^{-2} \mathrm{~s}^{-1}\right)$, using the web-based tool PDR Toolbox ${ }^{24}$ (Kaufman et al. 2006; Pound $\&$ Wolfire 2008). We set constraints using three line ratios: [O I]63/[C II], [O I $] 145 /\left[\mathrm{C} \mathrm{II}\right.$, and ([O I $\left.63+\left[\mathrm{C}_{\mathrm{II}}\right]\right) / L_{\mathrm{FIR}}$, where $L_{\mathrm{FIR}}$ is the IR luminosity longward of $30 \mu \mathrm{m}$. We assume that all three lines trace PDRs, and that there are no differential extinction effects. We estimate $L_{\mathrm{FIR}}$ using the same methods as for $L_{\mathrm{IR}}$ (Table 1).

For the whole sample (Figure 16) we find (taking a conservative cut of $\chi_{\text {red }}^{2}<5$ ) ranges of $10^{1}<n<10^{2.5}$ and $10^{2.2}<G_{0}<10^{3.6}$, with a power-law dependence between the two. The ranges of both $n$ and $G_{0}$ depend on optical class (Figure 17). For H II objects we find $10^{1.1}<n<10^{2.2}$ and $10^{2.4}<G_{0}<10^{3.3}$. For LINERS and Seyferts, however, the ranges widen: for LINERS we find $10^{0.8}<n<10^{2.5}$ and $10^{2.4}<G_{0}<10^{4.1}$, and for Sy2s we find $10^{0.7}<n<10^{3}$ and $10^{1.9}<G_{0}<10^{3.9}$. For Sy1s the range for $n$ is comparable to that of LINERs and Sy2s, but the range for $G_{0}$ increases to $10^{2.5}<G_{0}<10^{4.7}$. For Sy 1 s there is a secondary solution that is close to acceptable, which has $G_{0}$ and $n$ values approximately four orders of magnitude lower and higher, respectively, than the primary solution.

If we divide the sample in two by PAH 11.2 EW (top row of Figure 18), then we also see a difference. The range in $n$ for both samples is comparable at about $10^{0.8}<n<10^{2.5}$. However, the ranges for $G_{0}$ are different; for objects with prominent PAHs it is $10^{2.1}<G_{0}<10^{3.7}$, while for objects with weak PAHs it is $10^{2.6}<G_{0}<10^{4.3}$. We obtain similar ranges for both parameters if we instead divide the sample by merger stage (bottom row of Figure 18). This is consistent with a more intense ISRF destroying PAH molecules (see also e.g., Hernán-Caballero et al. 2009). It is, however, also consistent with a luminous AGN (with a harder UV radiation field) arising after the star formation has faded. This would give the same observation but with no direct relation between the two phenomena.

There are three caveats when using these models to estimate $G_{0}$ and $n$ for our sample. First, we cannot account for

24 http://dustem.astro.umd.edu/pdrt/

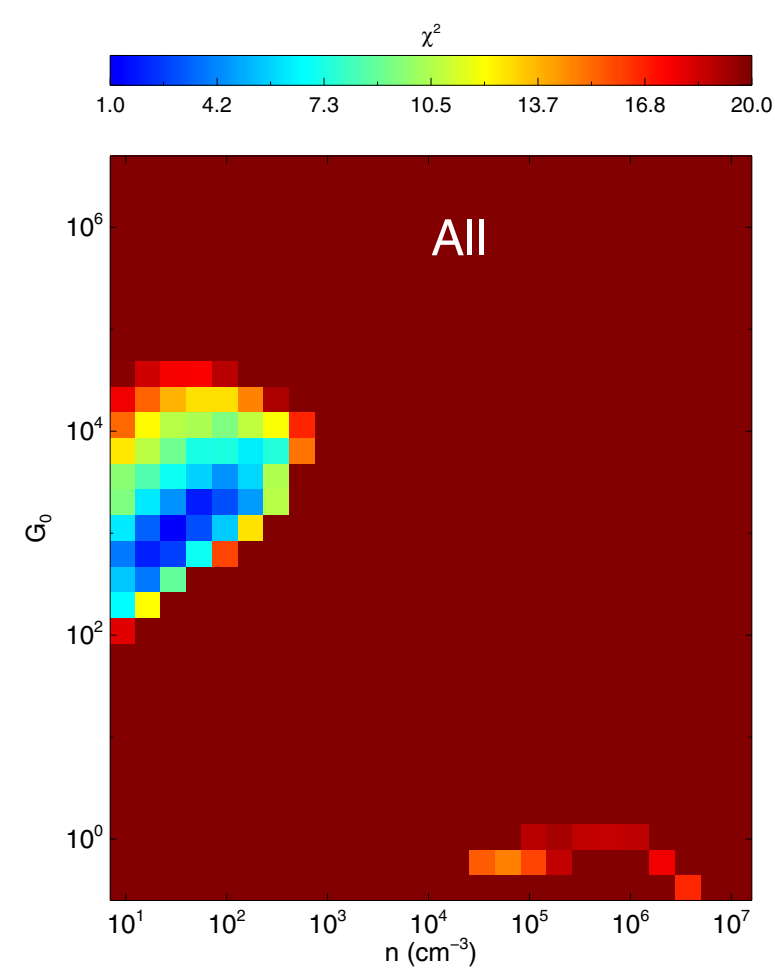

Figure 16. Results from simultaneous modeling of the $[\mathrm{OI}] 63 /[\mathrm{C} \mathrm{II}]$, [O I145/C II 158], and ([O I]63+[C II])/LFIR ratios, using PDRToolbox (Kaufman et al. 2006; Pound \& Wolfire 2008), to constrain the electron density $n$ and incident far-UV radiation field intensity $G_{0}$ (Section 3.6). The image is the median-combined stacked $n$ vs. $G_{0}$ plane for the whole sample. Units of $n$ are $\mathrm{cm}^{-3}$ and units of $G_{0}$ are the local Galactic interstellar FUV field found by Habing $1968\left(1.6 \times 10^{-3} \mathrm{erg} \mathrm{cm}^{-2} \mathrm{~s}^{-1}\right)$. The $x$ and $y$ axis ranges are fixed by PDRToolbox

(A color version of this figure is available in the online journal.)

different beam filling factors for different lines. This is potentially a significant problem for [C II] (see Sections 3.1.2 and 3.2.3). Second, these models have difficulty in predicting PAH emission strengths (Luhman et al. 2003; Abel et al. 2009), suggesting an incomplete description of the dependence of farIR line strengths on dust-grain size distribution, PAH properties, and ISRF spectral shape (see also, e.g., Okada et al. 2013). Since our targets are dusty, it is surprising that we obtain reasonable solutions, indicating that the line ratios and the adopted IR luminosities are compatible with each other. Our derived parameter ranges for $G_{0}$ and $n$, however, should be viewed with caution.

The third caveat is that the $\left[\mathrm{O}_{\mathrm{I}}\right]$ lines are complex to model. Assuming emission in PDRs, then, like [C II], the [O I] lines are expected to form within $A_{v} \sim 3$ magnitudes of the PDR surfaces. It is in these regions that all of the carbon and oxygen should be ionized and atomic, respectively, with gas temperatures between about 250 and $700 \mathrm{~K}$ (e.g., Kaufman et al. 1999). The dust in these regions has only a small effect on [C II], but can have a large impact on the [O I] levels, which are affected by both radiative and collisional processes. Such processes can alter the power of [O I]63 via the absorption of $63 \mu \mathrm{m}$ lineemitted photons by dust grains, or by the pumping of oxygen atoms by $63 \mu \mathrm{m}$ continuum dust emitted photons. The effect of dust should not be neglected when modeling [O I]63, or the [O I] line ratio, in sources that are optically thick at wavelengths shorter than $100 \mu \mathrm{m}$ (González-Alfonso et al. 2008). 

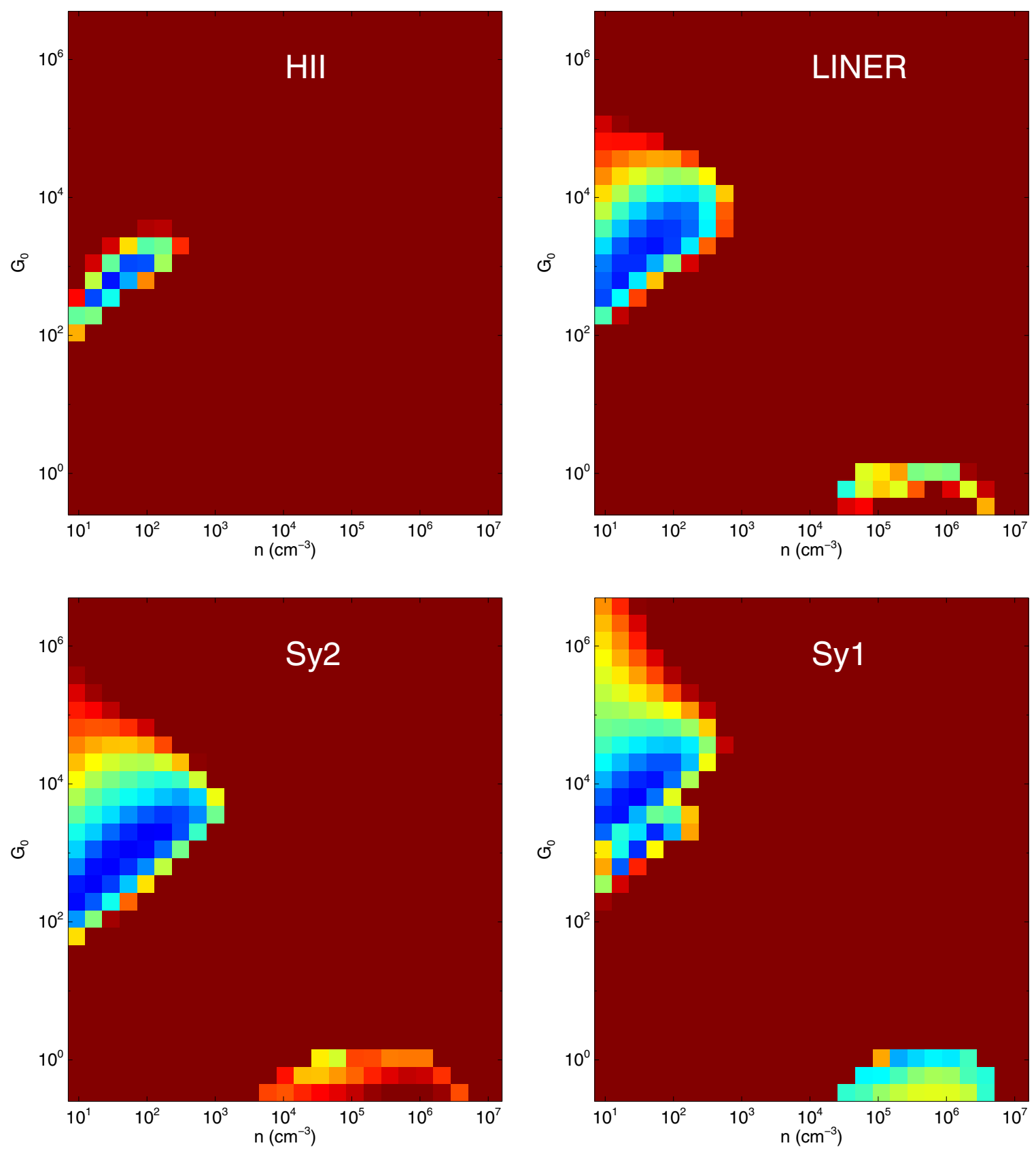

Figure 17. Results from PDRToolbox modeling, see Figure 16 for details. The panels show subsamples divided on optical spectral type.

(A color version of this figure is available in the online journal.)

\subsection{Merger Stage}

There is evidence that the power source in ULIRGs evolves as a function of merger stage, with star formation dominating, on average, until the progenitors coalesce, whereupon an AGN sometimes becomes energetically important (e.g., Rigopoulou et al. 1999; Farrah et al. 2009). We may therefore see a correlation between far-IR line properties and merger stage. However, we find no correlations between merger stage and far-IR line luminosities. We also see no trend with any far-IR line ratio. Comparing merger stage to normalized far-IR line luminosities (Figure 19), there may be a weak trend; for [O I]63 and longer lines, the advanced mergers might show a smaller normalized line luminosity than earlier stage mergers. This is consistent with the line luminosities tracing star formation, and with star formation becoming less important as merger stage advances. However, the trend is not strong and does not depend on optical classification or PAH $11.2 \mu \mathrm{m}$ EW.

\subsection{SMBH Mass}

Scaling relations have been derived between the masses of supermassive black holes and the FWHM and continuum luminosities of several UV, optical, and mid-IR emission lines (Kaspi et al. 2000; Vestergaard 2002; Vestergaard \& Peterson 2006; Dasyra et al. 2008). Here, using SMBH masses derived from optical lines (Table 1), we explore whether or not there exist correlations between far-IR line properties and SMBH mass. While the absolute uncertainties on the SMBH masses from these studies are of the order of $0.4 \mathrm{dex}$, the relative uncertainties within the sample are likely smaller as we focus on one class 

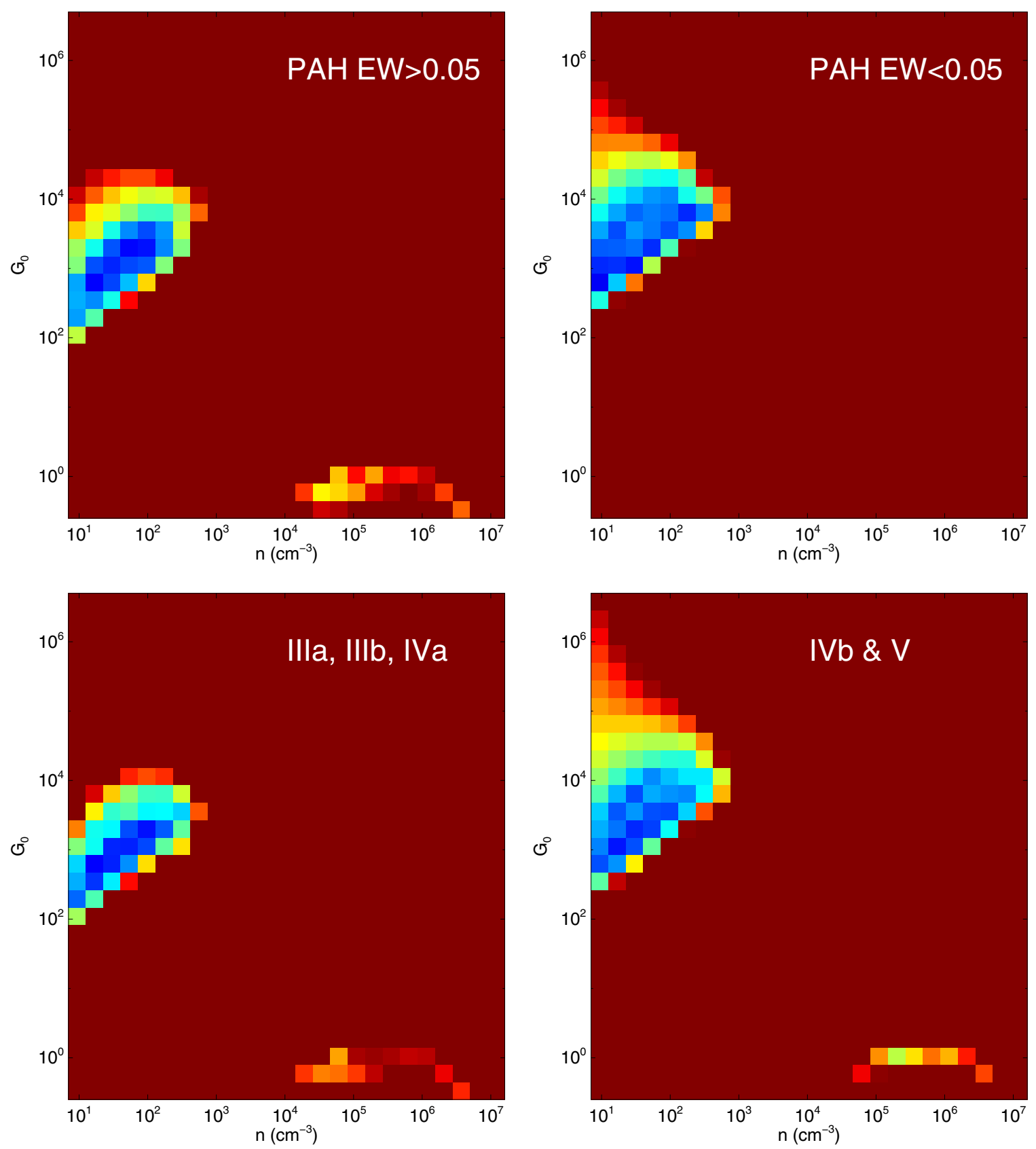

Figure 18. Results from PDRToolbox modeling, see Figure 16 for details. The top row shows samples divided on PAH $11.2 \mu \mathrm{m}$ EW $(<0.05 \mu \mathrm{m}$ vs. $\geqslant 0.05 \mu \mathrm{m})$. The bottom row shows samples divided on merger stage (Table 1).

(A color version of this figure is available in the online journal.)

of object and use $\mathrm{H} \beta$ derived masses in nearly all cases. We therefore assume an error on SMBH mass of $20 \%$.

We compare the SMBH masses to line luminosities in Figure 20. For the whole sample, no line shows a trend with SMBH mass. Considering only the Sy1s and Sy2s and excluding $3 \mathrm{C} 273$, some line luminosities qualitatively show a positive trend with SMBH mass. The trend is only significant for $L_{[\mathrm{NIII}]}$, for which we derive

$$
\begin{aligned}
\log _{10}\left(\frac{M_{\mathrm{SMBH}}}{M_{\odot}}\right)= & (1.09 \pm 1.43) \\
& +(0.82 \pm 0.18) \log _{10}\left(\frac{L_{[\mathrm{NIII}]}}{L_{\odot}}\right) .
\end{aligned}
$$

We see similar results if we instead compare $M_{\mathrm{SMBH}} / L_{\mathrm{IR}}$ to $L_{\text {Line }} / L_{\text {IR }}$.

It is plausible to exclude $3 \mathrm{C} 273$, since it is the only blazar in the sample. We do not, however, claim that this relation is real for four reasons. First, if we assume an (still reasonable) error on the SMBH masses in excess of $30 \%$, then the relation is no longer significant. Second, there is no trend of $L_{[\mathrm{N} \text { III] }}$ with the AGN diagnostics considered in Section 3.5. Third, if this relation is real, then it is strange that we do not see a correlation of SMBH mass with $L_{[\mathrm{O} \text { III] }}$ (see Table 2, though there is a potentially important difference; [N III] is a groundstate transition, whereas [O III] is not). Fourth, we searched for correlations between SMBH mass and far-IR continuum luminosities near $57 \mu \mathrm{m}$, but did not find any clear relations. 


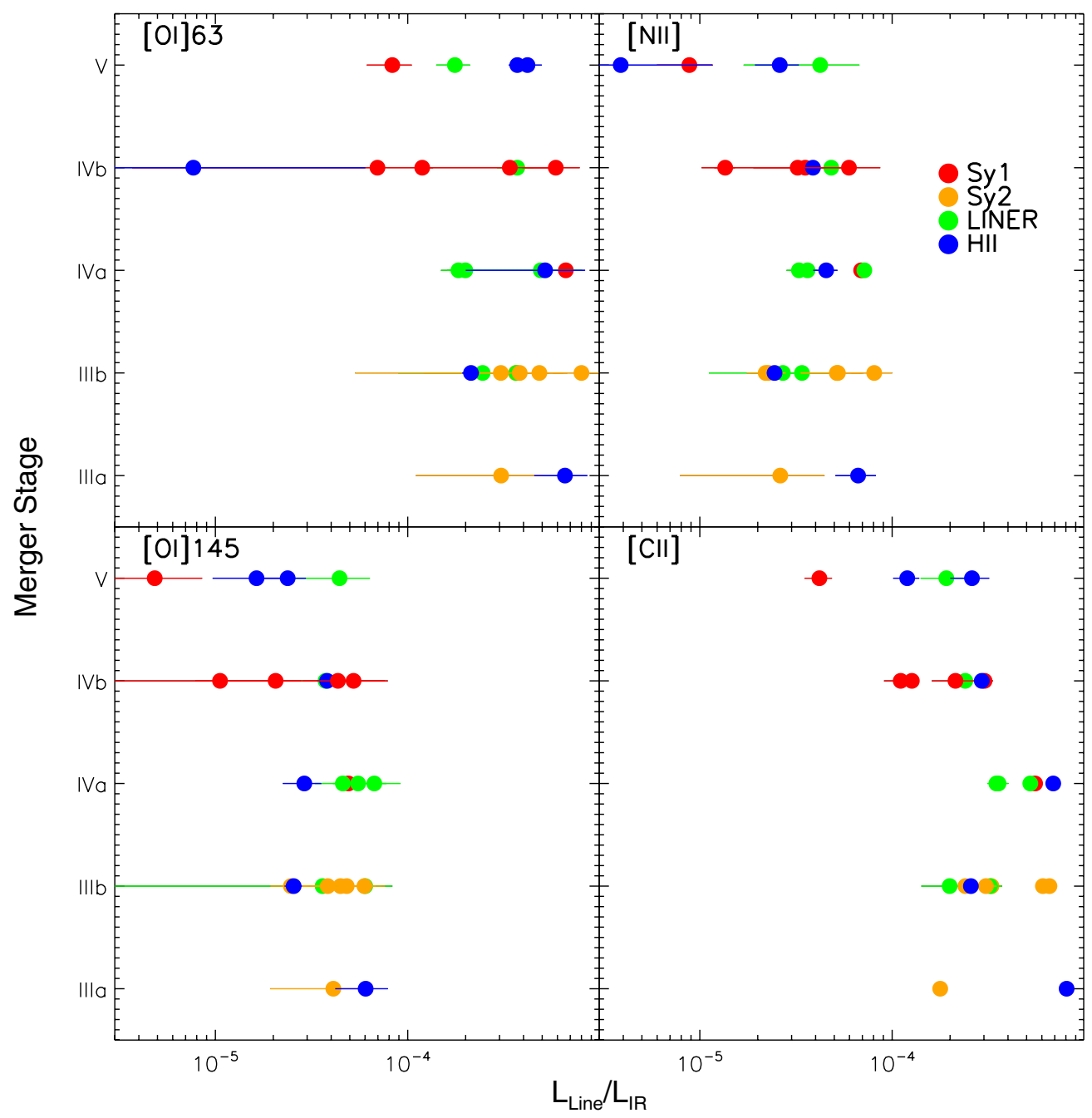

Figure 19. Line luminosities normalized by $L_{\mathrm{IR}}$, vs. merger stage (Section 3.7).

(A color version of this figure is available in the online journal.)

\section{CONCLUSIONS}

We have presented observations with PACS on board Herschel of 25 ULIRGs at $z<0.27$. We observed each ULIRG in six lines: [O III]52 $\mu \mathrm{m},\left[\mathrm{N}_{\mathrm{III}}\right] 57 \mu \mathrm{m},\left[\mathrm{O}_{\mathrm{I}}\right] 63 \mu \mathrm{m}$, [N II] $122 \mu \mathrm{m},\left[\mathrm{O}_{\mathrm{I}}\right] 145 \mu \mathrm{m}$, and [C II]158 $\mu \mathrm{m}$. We used the properties of these lines, together with diagnostics at other wavelengths, to draw the following conclusions.

1. In most cases, the line profiles are reproducible by single Gaussians, with widths between $250 \mathrm{~km} \mathrm{~s}^{-1}$ and $600 \mathrm{~km} \mathrm{~s}^{-1}$. The exceptions are [O I]63 and [C II], which occasionally show self absorption and a second, broad component, respectively. We do not see significant systemic offsets of the far-IR lines compared to the optical redshifts. The line luminosities range from just under $10^{7} L_{\odot}$ to just over $2 \times 10^{9} L_{\odot}$. The [O I]63 and [C II] lines are usually the most luminous, while [O I] 145 and [N II] are usually the least luminous. The line luminosities correlate with each other, though in no case is the correlation particularly strong. Simple line ratio diagnostics suggest relatively low gas densities, on average, and that a significant fraction of the $\left[\mathrm{C}_{\mathrm{II}}\right]$ emission originates from outside $\mathrm{H}$ II regions.

2. There is a deficit in the $\left[\mathrm{O}_{\mathrm{I}}\right] 63 / L_{\mathrm{IR}},[\mathrm{N} \mathrm{II}] / L_{\mathrm{IR}}$, [O I $] 145 / L_{\mathrm{IR}}$, and $\left[\mathrm{C}_{\mathrm{II}}\right] / L_{\mathrm{IR}}$ ratios compared to lower luminosity systems, of factors of $2.75,4.46,1.50$, and 4.95 , respectively. There is evidence that the $\left[\mathrm{C}_{\mathrm{II}}\right]$ and [N II] deficits correlate with $9.7 \mu \mathrm{m}$ silicate feature strength $\left(S_{\mathrm{Sil}}\right)$; if $S_{\mathrm{Sil}} \gtrsim 1.4$, then the [C II] and [N II] deficits rise with increasing $S_{\mathrm{Sil}}$. We also see a correlation between $\left[L_{\mathrm{PAH}}\right] / L_{\mathrm{IR}}$ and $S_{\mathrm{Sil}}$. Furthermore, the [C II] deficit correlates with merger stage; objects in advanced mergers show a greater deficit than objects in early stage mergers. These results are consistent with the majority of the line deficits arising due to increased levels of dust in $\mathrm{H}$ II regions. However, we propose that a significant fraction of the [C II] deficit arises from an additional mechanism, plausibly grain charging in PDRs and/or the diffuse ISM.

3. The line luminosities only weakly correlate with IR luminosity. The correlations improve if Sy1 objects are excluded. Doing so, and fitting a relation of the form $\log _{10}\left(L_{\mathrm{IR}}\right)=\alpha+\beta \log _{10}\left(L_{\text {Line }}\right)$, yields

$$
\log _{10}\left(\frac{L_{\mathrm{IR}}}{L_{\odot}}\right)=(4.46 \pm 1.77)+(0.92 \pm 0.20) \log _{10}\left(L_{[\mathrm{O} \mathrm{III]}}\right)
$$

$$
=(4.74 \pm 1.59)+(0.94 \pm 0.19) \log _{10}\left(L_{[\mathrm{N} \mathrm{III]}}\right)
$$




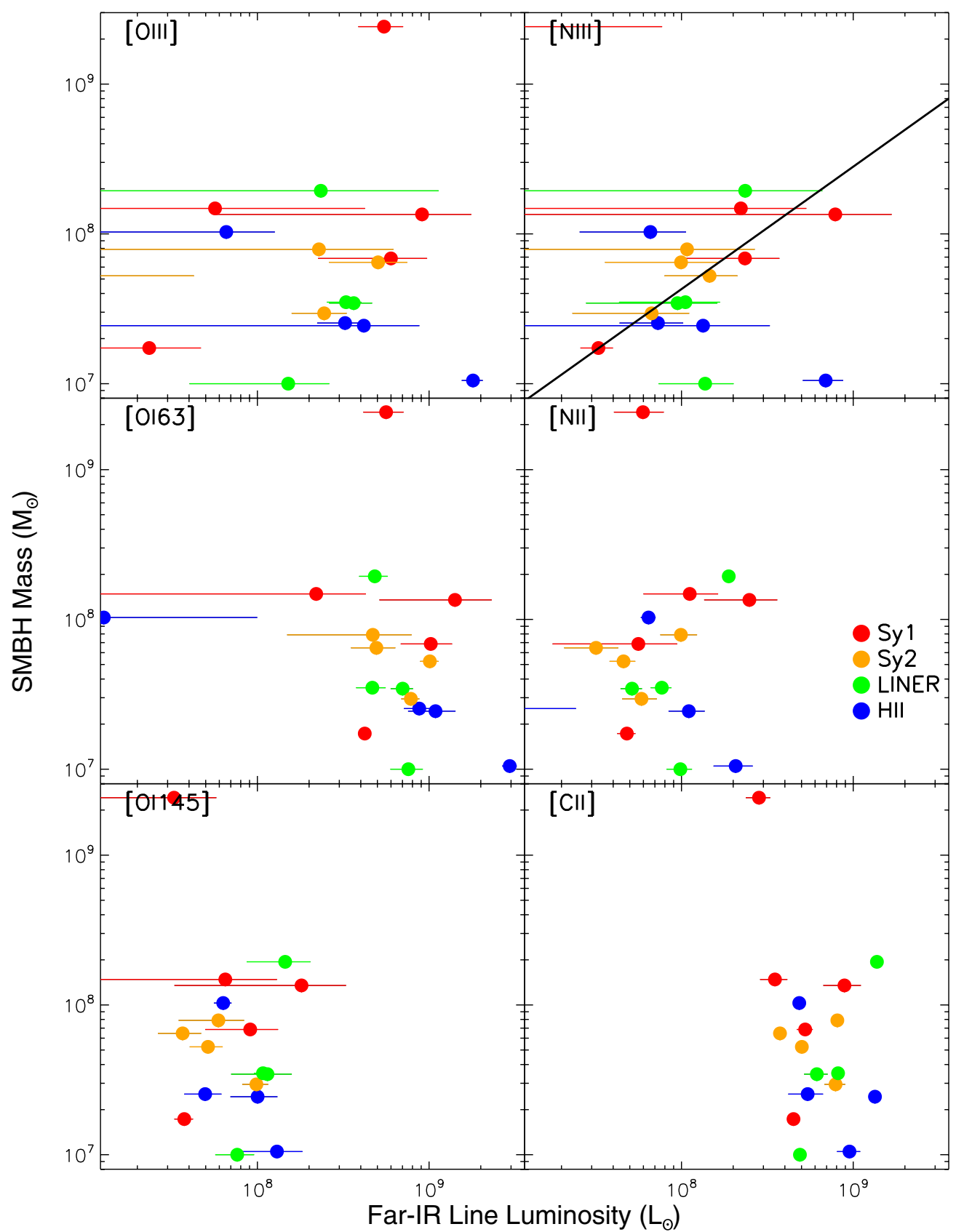

Figure 20. Far-IR line luminosities vs. SMBH mass (Section 3.8). The fit in the [N III] panel is Equation (9), and is to the Seyferts, excluding $3 \mathrm{C} 273$.

(A color version of this figure is available in the online journal.)

$$
\begin{aligned}
& =(4.28 \pm 1.89)+(0.91 \pm 0.21) \log _{10}\left(L_{[\mathrm{O} I 63}\right) \\
& =(5.29 \pm 1.57)+(0.89 \pm 0.20) \log _{10}\left(L_{[\mathrm{NII}}\right) \\
& =(1.75 \pm 2.11)+(1.34 \pm 0.27) \log _{10}\left(L_{[\mathrm{O} \mathrm{I}] 145}\right) \\
& =(6.73 \pm 2.44)+(0.64 \pm 0.27) \log _{10}\left(L_{[\mathrm{C} \mathrm{II}]}\right)
\end{aligned}
$$

The best tracers of $L_{\mathrm{IR}}$ are thus the five shorter wavelength lines. The [C II] line is a poor tracer of $L_{\mathrm{IR}}$, which is accurate to about an order of magnitude at best. Its accuracy does not noticeably improve if objects with a strong [C II] deficit are excluded.
4. The continuum luminosity densities near the wavelengths of the lines correlate with $L_{\mathrm{IR}}$, irrespective of the presence of Sy1s. We derive

$$
\begin{aligned}
\log _{10}\left(\frac{L_{\text {IR }}}{L_{\odot}}\right)= & (12.87 \pm 0.09) \\
& +(0.88 \pm 0.17) \log _{10}\left(\frac{L_{52 \mu \mathrm{m}}}{L_{\odot} \mathrm{Hz}^{-1}}\right) \\
= & (12.86 \pm 0.05)+(0.91 \pm 0.11) \log _{10}\left(L_{57 \mu \mathrm{m}}\right) \\
= & (12.86 \pm 0.08)+(0.98 \pm 0.18) \log _{10}\left(L_{63 \mu \mathrm{m}}\right) \\
= & (12.95 \pm 0.07)+(0.87 \pm 0.11) \log _{10}\left(L_{122 \mu \mathrm{m}}\right)
\end{aligned}
$$




$$
=(13.05 \pm 0.11)+(0.90 \pm 0.16) \log _{10}\left(L_{145 \mu \mathrm{m}}\right)
$$

$$
=(13.08 \pm 0.11)+(0.83 \pm 0.11) \log _{10}\left(L_{158 \mu \mathrm{m}}\right) .
$$

5. We find correlations between star formation rate, estimated using $L_{\mathrm{PAH}}$, and both line luminosities and continuum luminosity densities. For line luminosities, we derive

$$
\begin{aligned}
& \log _{10}\left(\frac{\dot{M}_{\odot}}{M_{\odot} \mathrm{yr}^{-1}}\right)=(-7.02 \pm 1.25) \\
&+(1.07 \pm 0.14) \log _{10}\left(L_{[\mathrm{O} \mathrm{III}]}\right) \\
&=(-5.13 \pm 0.72)+(0.91 \pm 0.09) \log _{10}\left(L_{[\mathrm{N} \mathrm{III]}]}\right)(-5.44 \pm 1.79)+(0.86 \pm 0.20) \log _{10}\left(L_{[\mathrm{O} \mathrm{I}] 63}\right) \\
&=(-7.30 \pm 0.87)+(1.19 \pm 0.11) \log _{10}\left(L_{[\mathrm{NII}]}\right) \\
&=(-10.04 \pm 1.34)+(1.55 \pm 0.17) \log _{10}\left(L_{[\mathrm{O} \mathrm{I}] 145}\right) \\
&=(-6.24 \pm 1.72)+(0.95 \pm 0.19) \log _{10}\left(L_{[\mathrm{CII}]}\right),
\end{aligned}
$$

while for the continuum luminosity densities we derive

$$
\begin{aligned}
& \log _{10}\left(\dot{M}_{\odot}\right)=(3.24 \pm 0.24)+(1.89 \pm 0.46) \log _{10}\left(L_{52 \mu \mathrm{m}}\right) \\
& =(2.95 \pm 0.10)+(1.38 \pm 0.19) \log _{10}\left(L_{57 \mu \mathrm{m}}\right) \\
& =(3.04 \pm 0.17)+(1.79 \pm 0.39) \log _{10}\left(L_{63 \mu \mathrm{m}}\right) \\
& =(2.83 \pm 0.11)+(0.93 \pm 0.16) \log _{10}\left(L_{122 \mu \mathrm{m}}\right) \\
& =(3.02 \pm 0.18)+(1.09 \pm 0.25) \log _{10}\left(L_{145} \mu \mathrm{m}\right) \\
& =(3.36 \pm 0.22)+(1.42 \pm 0.30) \log _{10}\left(L_{158 \mu \mathrm{m}}\right)
\end{aligned}
$$

On average, the shorter wavelength continua show stronger correlations.

6. Assuming the $[\mathrm{O} \mathrm{I}]$ and $[\mathrm{C} I \mathrm{II}$ lines arise mainly in PDRs, we use a simple model to extract estimates for the hydrogen nucleus density, $n$, and incident far-ultraviolet radiation field, $G_{0}$, in the far-IR line emitting gas. We find $10^{1}<n<$ $10^{2.5}$ and $10^{2.2}<G_{0}<10^{3.6}$ for the whole sample, with a power-law dependence between the two. The ranges depend on optical spectral class; for H II-like objects we find $10^{1.1}<$ $n<10^{2.2}$ and $10^{2.4}<G_{0}<10^{3.3}$, while for Sy1s we find $10^{0.8}<n<10^{2.7}$ and $10^{2.5}<G_{0}<10^{4.7}$. There is also a dependence of $G_{0}$ on the importance of star formation; objects with weak PAHs have $10^{2.6}<G_{0}<10^{4.3}$, while objects with prominent PAHs have $10^{2.1}<G_{0}<10^{3.7}$. We find similar ranges for early- versus late-stage mergers. This is consistent with, but not exclusively supportive of, a more intense ISRF destroying PAH molecules.
7. We searched for relations between far-IR line luminosities and ratios, and several other parameters: AGN activity (either from optical spectral class or the detection of [Ne v]14.32), merger stage, mid-IR excitation, and SMBH mass. For the first three parameters we found no relations. We conclude that the far-IR lines do not arise primarily due to AGN activity and that the properties of the farIR line emitting gas do not strongly depend on either mid-IR excitation or merger stage. For SMBH mass we found one superficially striking correlation, with $L_{[\mathrm{N} \mathrm{III]}}$, but subsequent tests were not supportive. We conclude that farIR line luminosities do not straightforwardly trace SMBH mass.

We thank the staff of the Herschel helpdesk for many valuable discussions, and the referee for a very helpful report. Herschel is an ESA space observatory with science instruments provided by European-led Principal Investigator consortia and with important participation from NASA. This work is based on observations made with the Spitzer Space Telescope. Spitzer is operated by the Jet Propulsion Laboratory, California Institute of Technology under a contract with NASA. This research has made extensive use of the NASA/IPAC Extragalactic Database (NED), which is operated by the Jet Propulsion Laboratory, California Institute of Technology, under contract with NASA, and of NASA's Astrophysics Data System. This research has also made use of Ned Wright's online cosmology calculator (Wright 2006). V.L. is supported by a CEA/Marie Curie Eurotalents fellowship. J.A. acknowledges support from the Science and Technology Foundation (FCT, Portugal) through the research grants PTDC/CTE-AST/105287/2008, PEst-OE/FIS/UI2751/2011, and PTDC/FIS-AST/2194/2012. E.G.-A. is a Research Associate at the Harvard-Smithsonian Center for Astrophysics and thanks the support by the Spanish Ministerio de Economía y Competitividad under projects AYA2010-21697-C05-0 and FIS2012-39162-C06-01.

Facilities: Herschel, Spitzer

\section{REFERENCES}

Abel, N. P., Dudley, C., Fischer, J., Satyapal, S., \& van Hoof, P. A. M. 2009, ApJ, 701,1147

Abel, N. P., Sarma, A. P., Troland, T. H., \& Ferland, G. J. 2007, ApJ, 662, 1024 Armus, L., Charmandaris, V., Bernard-Salas, J., et al. 2007, ApJ, 656, 148 Austermann, J. E., Dunlop, J. S., Perera, T. A., et al. 2010, MNRAS, 401, 160 Bernard-Salas, J., Habart, E., Arab, H., et al. 2012, A\&A, 538, A37

Bernard-Salas, J., Peeters, E., Sloan, G. C., et al. 2006, ApJL, 652, L29

Bernard-Salas, J., Pottasch, S. R., Beintema, D. A., \& Wesselius, P. R. 2001, A\&A, 367, 949

Bernard-Salas, J., Spoon, H. W. W., Charmandaris, V., et al. 2009, ApJS, 184,230

Berta, S., Lonsdale, C. J., Siana, B., et al. 2007, A\&A, 467, 565

Blommaert, J. A. D. L., Cami, J., Szczerba, R., \& Barlow, M. J. 2005, SSRv, 119,215

Borys, C., Blain, A. W., Dey, A., et al. 2006, ApJ, 636, 134

Borys, C., Chapman, S., Halpern, M., \& Scott, D. 2003, MNRAS, 344, 385

Boselli, A., Gavazzi, G., Lequeux, J., \& Pierini, D. 2002, A\&A, 385, 454

Brandl, B. R., Bernard-Salas, J., Spoon, H. W. W., et al. 2006, ApJ, 653, 1129

Brauher, J. R., Dale, D. A., \& Helou, G. 2008, ApJS, 178, 280

Bridge, C. R., Appleton, P. N., Conselice, C. J., et al. 2007, ApJ, 659, 931

Bridge, C. R., Blain, A., Borys, C. J. K., et al. 2013, ApJ, 769, 91

Bushouse, H. A., Borne, K. D., Colina, L., et al. 2002, ApJS, 138, 1

Calzetti, D., Kennicutt, R. C., Engelbracht, C. W., et al. 2007, ApJ, 666, 870

Chapman, S. C., Windhorst, R., Odewahn, S., Yan, H., \& Conselice, C. 2003, ApJ, 599, 92

Clements, D. L., Sutherland, W. J., McMahon, R. G., \& Saunders, W. 1996, MNRAS, 279, 477

Combes, F., García-Burillo, S., Braine, J., et al. 2011, A\&A, 528, A124 
Combes, F., García-Burillo, S., Braine, J., et al. 2013, A\&A, 550, A41

Coppin, K. E. K., Danielson, A. L. R., Geach, J. E., et al. 2012, MNRAS, 427, 520

Cui, J., Xia, X.-Y., Deng, Z.-G., Mao, S., \& Zou, Z.-L. 2001, AJ, 122, 63

Dale, D. A., Smith, J. D. T., Armus, L., et al. 2006, ApJ, 646, 161

Dasyra, K. M., Ho, L. C., Armus, L., et al. 2008, ApJL, 674, L9

Dasyra, K. M., Tacconi, L. J., Davies, R. I., et al. 2006, ApJ, 651, 835

de Graauw, T., Helmich, F. P., Phillips, T. G., et al. 2010, A\&A, 518, L6

de Looze, I., Baes, M., Bendo, G. J., Cortese, L., \& Fritz, J. 2011, MNRAS, 416, 2712

Desai, V., Armus, L., Spoon, H. W. W., et al. 2007, ApJ, 669, 810

Diaz-Santos, T., Armus, L., Charmandaris, V., et al. 2013, ApJ, 774, 68

Dole, H., Gispert, R., Lagache, G., et al. 2001, A\&A, 372, 364

Draper, A. R., \& Ballantyne, D. R. 2012, ApJL, 753, L37

Efstathiou, A., Pearson, C., Farrah, D., et al. 2013, MNRAS, submitted

Farrah, D., Afonso, J., Efstathiou, A., et al. 2003, MNRAS, 343, 585

Farrah, D., Bernard-Salas, J., Spoon, H. W. W., et al. 2007a, ApJ, 667, 149

Farrah, D., Connolly, B., Connolly, N., et al. 2009, ApJ, 700, 395

Farrah, D., Lacy, M., Priddey, R., Borys, C., \& Afonso, J. 2007b, ApJL, 662, L59

Farrah, D., Lonsdale, C. J., Weedman, D. W., et al. 2008, ApJ, 677, 957

Farrah, D., Rowan-Robinson, M., Oliver, S., et al. 2001, MNRAS, 326, 1333

Farrah, D., Surace, J. A., Veilleux, S., Sanders, D. B., \& Vacca, W. D. 2005, ApJ, 626, 70

Farrah, D., Verma, A., Oliver, S., Rowan-Robinson, M., \& McMahon, R. 2002, MNRAS, 329, 605

Fischer, J., Luhman, M. L., Satyapal, S., et al. 1999, Ap\&SS, 266, 91

Fischer, J., Sturm, E., González-Alfonso, E., et al. 2010, A\&A, 518, L41

Förster Schreiber, N. M., Roussel, H., Sauvage, M., \& Charmandaris, V. 2004, A\&A, 419, 501

Franceschini, A., Braito, V., Persic, M., et al. 2003, MNRAS, 343, 1181

Geach, J. E., Hickox, R. C., Diamond-Stanic, A. M., et al. 2013, ApJL, 767, L17

Gehrz, R. 1989, in IAU Symp. 135, Interstellar Dust, ed. L. J. Allamandola \& A. G. G. M. Tielens (Dordrecht: Kluwer), 445

Genzel, R., Lutz, D., Sturm, E., et al. 1998, ApJ, 498, 579

Genzel, R., Tacconi, L. J., Rigopoulou, D., Lutz, D., \& Tecza, M. 2001, ApJ, 563,527

González-Alfonso, E., Fischer, J., Bruderer, S., et al. 2013, A\&A, 550, A25

González-Alfonso, E., Fischer, J., Isaak, K., et al. 2010, A\&A, 518, L43

González-Alfonso, E., Smith, H. A., Ashby, M. L. N., et al. 2008, ApJ, 675, 303

Gorjian, V., Cleary, K., Werner, M. W., \& Lawrence, C. R. 2007, ApJL, 655, L73

Goto, T., Arnouts, S., Inami, H., et al. 2011, MNRAS, 410, 573

Graciá-Carpio, J., Sturm, E., Hailey-Dunsheath, S., et al. 2011, ApJL, 728, L7

Greene, J. E., \& Ho, L. C. 2007, ApJ, 667, 131

Griffin, M. J., Abergel, A., Abreu, A., et al. 2010, A\&A, 518, L3

Habing, H. J. 1968, BAN, 19, 421

Habing, H. J. 1996, A\&ARv, 7, 97

Hailey-Dunsheath, S., Sturm, E., Fischer, J., et al. 2012, ApJ, 755, 57

Hanami, H., Ishigaki, T., Fujishiro, N., et al. 2012, PASJ, 64, 70

Hernán-Caballero, A., Pérez-Fournon, I., Hatziminaoglou, E., et al. 2009, MNRAS, 395, 1695

Ho, L. C., \& Keto, E. 2007, ApJ, 658, 314

Hou, L. G., Han, J. L., Kong, M. Z., \& Wu, X.-B. 2011, ApJ, 732, 72

Houck, J. R., Roellig, T. L., van Cleve, J., et al. 2004, ApJS, 154, 18

Huang, J.-S., Faber, S. M., Daddi, E., et al. 2009, ApJ, 700, 183

Hwang, H. S., Elbaz, D., Lee, J. C., et al. 2010, A\&A, 522, A33

Iglesias-Páramo, J., Buat, V., Hernández-Fernández, J., et al. 2007, ApJ, 670,279

Imanishi, M., Dudley, C. C., Maiolino, R., et al. 2007, ApJS, 171, 72

Johnson, S. P., Wilson, G. W., Wang, Q. D., et al. 2013, MNRAS, 431, 662

Kartaltepe, J. S., Sanders, D. B., Le Floc'h, E., et al. 2010, ApJ, 721, 98

Kaspi, S., Smith, P. S., Netzer, H., et al. 2000, ApJ, 533, 631

Kaufman, M. J., Wolfire, M. G., \& Hollenbach, D. J. 2006, ApJ, 644, 283

Kaufman, M. J., Wolfire, M. G., Hollenbach, D. J., \& Luhman, M. L. 1999, ApJ, 527,795

Kawakatu, N., Anabuki, N., Nagao, T., Umemura, M., \& Nakagawa, T. 2006, ApJ, 637, 104

Kawakatu, N., Imanishi, M., \& Nagao, T. 2007, ApJ, 661, 660

Khramtsova, M. S., Wiebe, D. S., Boley, P. A., \& Pavlyuchenkov, Y. N. 2013, MNRAS, 431, 2006

Laurent, O., Mirabel, I. F., Charmandaris, V., et al. 2000, A\&A, 359, 887

Lebouteiller, V., Barry, D. J., Spoon, H. W. W., et al. 2011, ApJS, 196, 8

Lebouteiller, V., Bernard-Salas, J., Sloan, G. C., \& Barry, D. J. 2010, PASP, 122,231

Lebouteiller, V., Cormier, D., Madden, S. C., et al. 2012, A\&A, 548, A91
Le Floc'h, E., Papovich, C., Dole, H., et al. 2005, ApJ, 632, 169

Levenson, N. A., Sirocky, M. M., Hao, L., et al. 2007, ApJL, 654, L45

Lo Faro, B., Franceschini, A., Vaccari, M., et al. 2013, ApJ, 762, 108

Lonsdale, C. J., Farrah, D., \& Smith, H. E. 2006, in Ultraluminous Infrared Galaxies, Astrophysics Update 2-Topical and Timely Reviews on Astronomy and Astrophysics, ed. J. W. Mason (Chichester: Praxis), 285

Lonsdale, C. J., Polletta, M. d. C., Omont, A., et al. 2009, ApJ, 692, 422

Luhman, M. L., Satyapal, S., Fischer, J., et al. 2003, ApJ, 594, 758

Lutz, D., Kunze, D., Spoon, H. W. W., \& Thornley, M. D. 1998, A\&A, 333, L75

Madden, S. C., Galliano, F., Jones, A. P., \& Sauvage, M. 2006, A\&A, 446, 877

Magdis, G. E., Elbaz, D., Hwang, H. S., et al. 2010, MNRAS, 409, 22

Magnelli, B., Lutz, D., Santini, P., et al. 2012, A\&A, 539, A155

Malhotra, S., Kaufman, M. J., Hollenbach, D., et al. 2001, ApJ, 561, 766

Melbourne, J., Ammons, M., Wright, S. A., et al. 2008, AJ, 135, 1207

Meng, X.-M., Wu, H., Gu, Q.-S., Wang, J., \& Cao, C. 2010, ApJ, 718, 928

Meusinger, H., Stecklum, B., Theis, C., \& Brunzendorf, J. 2001, A\&A, 379,845

Mortier, A. M. J., Serjeant, S., Dunlop, J. S., et al. 2005, MNRAS, 363, 563

Nardini, E., \& Risaliti, G. 2011, MNRAS, 415, 619

Nardini, E., Risaliti, G., Watabe, Y., Salvati, M., \& Sani, E. 2010, MNRAS, 405, 2505

Negishi, T., Onaka, T., Chan, K.-W., \& Roellig, T. L. 2001, A\&A, 375, 566

Nenkova, M., Sirocky, M. M., Nikutta, R., Ivezić, Ž., \& Elitzur, M. 2008, ApJ, 685,160

Nordon, R., Lutz, D., Genzel, R., et al. 2012, ApJ, 745, 182

Okada, Y., Onaka, T., Shibai, H., \& Doi, Y. 2003, A\&A, 412, 199

Okada, Y., Pilleri, P., Berné, O., et al. 2013, A\&A, 553, A2

Oliva, E., Moorwood, A. F. M., Drapatz, S., Lutz, D., \& Sturm, E. 1999, A\&A, 343,943

Ott, S. 2010, in ASP Conf. Ser. 434, Astronomical Data Analysis Software and Systems XIX, ed. Y. Mizumoto, K.-I. Morita, \& M. Ohishi (San Francisco, CA: ASP), 139

Peeters, E., Martín-Hernández, N. L., Damour, F., et al. 2002, A\&A, 381, 571

Peeters, E., Spoon, H. W. W., \& Tielens, A. G. G. M. 2004, ApJ, 613, 986

Pilbratt, G. L., Riedinger, J. R., Passvogel, T., et al. 2010, A\&A, 518, L1

Planck Collaboration, Ade, P. A. R., Aghanim, N., et al. 2013, A\&A, submitted (arXiv:1303.5076)

Poglitsch, A., Herrmann, F., Genzel, R., et al. 1996, ApJL, 462, L43

Poglitsch, A., Waelkens, C., Geis, N., et al. 2010, A\&A, 518, L2

Pound, M. W., \& Wolfire, M. G. 2008, in ASP Conf. Ser. 394, Astronomical Data Analysis Software and Systems XVII, ed. R. W. Argyle, P. S. Bunclark, \& J. R. Lewis (San Francisco, CA: ASP), 654

Rigopoulou, D., Spoon, H. W. W., Genzel, R., et al. 1999, AJ, 118, 2625

Rodríguez Zaurín, J., Tadhunter, C. N., Rose, M., \& Holt, J. 2013, MNRAS, 432, 138

Rothberg, B., Fischer, J., Rodrigues, M., \& Sanders, D. B. 2013, ApJ, 767, 72

Rowan-Robinson, M., Mann, R. G., Oliver, S. J., et al. 1997, MNRAS, 289, 490

Rowan-Robinson, M., Roseboom, I. G., Vaccari, M., et al. 2010, MNRAS, 409, 2

Rupke, D. S. N., \& Veilleux, S. 2011, ApJL, 729, L27

Sajina, A., Yan, L., Fadda, D., Dasyra, K., \& Huynh, M. 2012, ApJ, 757, 13

Sanders, D. B., \& Mirabel, I. F. 1996, ARA\&A, 34, 749

Sargsyan, L., Lebouteiller, V., Weedman, D., et al. 2012, ApJ, 755, 171

Saunders, W., Sutherland, W. J., Maddox, S. J., et al. 2000, MNRAS, 317, 55

Shipley, H. V., Papovich, C., Rieke, G. H., et al. 2013, ApJ, 769, 75

Smail, I., Chapman, S. C., Blain, A. W., \& Ivison, R. J. 2004, ApJ, 616, 71

Smith, J. D. T., Draine, B. T., Dale, D. A., et al. 2007, ApJ, 656, 770

Smith, J. D. T., \& Houck, J. R. 2001, AJ, 121, 2115

Soifer, B. T., \& Neugebauer, G. 1991, AJ, 101, 354

Spaans, M., Tielens, A. G. G. M., van Dishoeck, E. F., \& Bakes, E. L. O. 1994, ApJ, 437, 270

Spinoglio, L., Malkan, M. A., Smith, H. A., González-Alfonso, E., \& Fischer, J. 2005, ApJ, 623, 123

Spinoglio, L., Tommasin, S., \& Malkan, M. A. 2009, RMxAA, 37, 120

Spoon, H. W. W., Armus, L., Marshall, J. A., et al. 2009, ApJ, 693, 1223

Spoon, H. W. W., Farrah, D., Lebouteiller, V., et al. 2013, ApJ, in press

Spoon, H. W. W., Marshall, J. A., Houck, J. R., et al. 2007, ApJL, 654, L49

Stacey, G. J., Hailey-Dunsheath, S., Ferkinhoff, C., et al. 2010, ApJ, 724, 957

Stierwalt, S., Armus, L., Surace, J. A., et al. 2013, ApJS, 206, 1

Sturm, E., González-Alfonso, E., Veilleux, S., et al. 2011, ApJL, 733, L16

Sturm, E., Lutz, D., Verma, A., et al. 2002, A\&A, 393, 821

Surace, J. A., Sanders, D. B., \& Evans, A. S. 2000, ApJ, 529, 170

Symeonidis, M., Page, M. J., \& Seymour, N. 2011, MNRAS, 411, 983

Symeonidis, M., Vaccari, M., Berta, S., et al. 2013, MNRAS, 431, 2317

Tacconi, L. J., Genzel, R., Lutz, D., et al. 2002, ApJ, 580, 73

Takata, T., Sekiguchi, K., Smail, I., et al. 2006, ApJ, 651, 713

Tran, Q. D., Lutz, D., Genzel, R., et al. 2001, ApJ, 552, 527 
Teng, S. H., \& Veilleux, S. 2010, ApJ, 725, 1848

Thornley, M. D., Schreiber, N. M. F., Lutz, D., et al. 2000, ApJ, 539, 641 Tielens, A. G. G. M. 2008, ARA\&A, 46, 289

Tremaine, S., Gebhardt, K., Bender, R., et al. 2002, ApJ, 574, 740

Vaccari, M., Marchetti, L., Franceschini, A., et al. 2010, A\&A, 518, L20

Valiante, E., Lutz, D., Sturm, E., et al. 2007, ApJ, 660, 1060

Vastel, C., Polehampton, E. T., Baluteau, J.-P., et al. 2002, ApJ, 581, 315

Vega, O., Clemens, M. S., Bressan, A., et al. 2008, A\&A, 484, 631

Veilleux, S., Kim, D.-C., Peng, C. Y., et al. 2006, ApJ, 643, 707

Veilleux, S., Kim, D.-C., \& Sanders, D. B. 2002, ApJS, 143, 315

Veilleux, S., Rupke, D. S. N., Kim, D.-C., et al. 2009, ApJS, 182, 628

Verma, A., Lutz, D., Sturm, E., et al. 2003, A\&A, 403, 829

Vestergaard, M. 2002, ApJ, 571, 733
Vestergaard, M., \& Peterson, B. M. 2006, ApJ, 641, 689

Wang, L., Farrah, D., Connolly, B., et al. 2011, MNRAS, 411, 1809

Wang, L., Farrah, D., Oliver, S. J., et al. 2013, MNRAS, 431, 648

Weedman, D. W., Hao, L., Higdon, S. J. U., et al. 2005, ApJ, 633, 706

Westmoquette, M. S., Clements, D. L., Bendo, G. J., \& Khan, S. A. 2012, MNRAS, 424, 416

Wright, E. L. 2006, PASP, 118, 1711

Xu, C. K., Zhao, Y., Scoville, N., et al. 2012, ApJ, 747, 85

Yuan, T.-T., Kewley, L. J., \& Sanders, D. B. 2010, ApJ, 709, 884

Zhang, S.-Y., Bian, W.-H., \& Huang, K.-L. 2008, A\&A, 488, 113

Zhao, Y., Lu, N., Xu, C. K., et al. 2013, ApJL, 765, L13

Zheng, X. Z., Xia, X. Y., Mao, S., Wu, H., \& Deng, Z. G. 2002, AJ, 124,18 